\title{
Injection therapy and denervation procedures for chronic low-back pain: a systematic review
}

\author{
Nicholas Henschke • Ton Kuijpers • Sidney M. Rubinstein • Marienke van Middelkoop • \\ Raymond Ostelo · Arianne Verhagen · Bart W. Koes • Maurits W. van Tulder
}

Received: 11 January 2010/Revised: 24 February 2010/Accepted: 11 April 2010/Published online: 29 April 2010

(C) The Author(s) 2010. This article is published with open access at Springerlink.com

\begin{abstract}
Injection therapy and denervation procedures are commonly used in the management of chronic low-back pain (LBP) despite uncertainty regarding their effectiveness and safety. To provide an evaluation of the current evidence associated with the use of these procedures, a systematic review was performed. Existing systematic reviews were screened, and the Cochrane Back Review Group trial register was searched for randomized controlled trials (RCTs) fulfilling the inclusion criteria. Studies were included if they recruited adults with chronic LBP, evaluated the use of injection therapy or denervation procedures and measured at least one clinically relevant outcome (such as pain or functional status). Two review authors independently assessed studies for eligibility and risk of bias (RoB). A meta-analysis was performed with clinically homogeneous studies, and the GRADE approach was used to determine
\end{abstract}

N. Henschke

The George Institute for International Health, Sydney, Australia

N. Henschke $(\bowtie) \cdot$ S. M. Rubinstein · R. Ostelo ·

M. W. van Tulder

EMGO+ Institute for Health and Care Research,

van der Boechhorststraat 7, 1081 BT Amsterdam,

The Netherlands

e-mail: n.henschke@vumc.nl

T. Kuijpers

Kwaliteitsinstituut voor de Gezondheidszorg CBO,

Utrecht, The Netherlands

M. van Middelkoop · A. Verhagen - B. W. Koes Department of General Practice, ErasmusMC,

University Medical Centre, Rotterdam, The Netherlands

R. Ostelo · M. W. van Tulder

Faculty of Earth and Life Sciences, VU University,

Amsterdam, The Netherlands the quality of evidence. In total, 27 RCTs were included, 14 on injection therapy and 13 on denervation procedures. 18 $(66 \%)$ of the studies were determined to have a low RoB. Because of clinical heterogeneity, only two comparisons could be pooled. Overall, there is only low to very low quality evidence to support the use of injection therapy and denervation procedures over placebo or other treatments for patients with chronic LBP. However, it cannot be ruled out that in carefully selected patients, some injection therapy or denervation procedures may be of benefit.

Keywords Injection - Back pain - Denervation . Review

\section{Introduction}

Chronic low-back pain (LBP) is related to disability and work absence and accounts for high social and health care costs in western societies [1]. The management of chronic LBP comprises a range of different intervention strategies including surgery, pharmacological interventions and nonmedical interventions such as exercise, behavioural therapy and alternative therapies. Non-surgical interventions, such as injection therapy and denervation procedures, have rapidly increasing rates of utilization and associated costs [2]. Injection therapy for chronic LBP involves injections of medications, irritants, or proteolytic enzymes into soft tissues outside or within the spine. Denervation procedures involve the application of various types of thermal or radiofrequency energy within the spine.

Over the past years, a substantial number of randomized clinical trials have been published on injection therapy and denervation procedures. In order to appropriately evaluate the effects of these interventions in a systematic review, it 
is important to make valid comparisons that are homogeneous with regard to study population, indications, interventions and different anatomical structures being targeted, controls, outcome measures and follow-up. Based on the available literature, this overview presents the current evidence on injections into and outside the spine and radiofrequency or thermal denervation procedures for nonspecific chronic LBP.

\section{Objectives}

The objective of this review was to determine the effectiveness of injection therapy (including injections into soft tissues outside or within the spine and chemonucleolysis), and radiofrequency or thermal denervation procedures for chronic LBP.

\section{Criteria for considering studies for this review}

Types of studies

Only randomized controlled trials with at least 1 day of follow-up were considered in this systematic review.

Types of participants

In order to be included in this review, participants of the randomized controlled trials (RCTs) must fulfil the following inclusion criteria: adult subjects ( $\geq 18$ years of age) with chronic ( $>12$ weeks duration) LBP (including subjects with radiculopathy or any other non-specific degenerative pathology, such as osteoarthritis). Exclusion criteria were (1) trials including subjects with specific LBP caused by pathological entities, such as vertebral spinal stenosis, ankylosing spondylitis, scoliosis, or coccydynia; (2) postpartum LBP or pelvic pain due to pregnancy; (3) postoperative studies; (4) prevention studies; and (5) abstracts or non-published studies.

\section{Types of interventions}

RCTs studying the following interventions were included in this overview: injection of medications or proteolytic enzymes (chemonucleolysis); and radiofrequency or thermal denervation procedures. All anatomical sites subject to injection therapy (intervertebral disc, facet joint, epidural space, intramuscular) and denervation procedures (facet joint, intervertebral disc, spinal nerves) for LBP were included. Additional interventions were allowed in all studies if there was a contrast for the injection therapy or denervation procedure in the study.
Types of outcome measures

To be included at least one of the following outcome measures should have been measured in the RCT: pain intensity [e.g. visual analog scale (VAS), numerical rating scale (NRS), McGill pain questionnaire], back specific functional status [e.g. Roland-Morris Disability Questionnaire, Oswestry Disability Index (ODI)], perceived recovery (e.g. overall improvement), and return to work (e.g. return to work status, sick leave days). The primary outcomes for this review were pain and functional status.

\section{Search methods for identification of studies}

Existing systematic reviews for the interventions were screened for studies fulfilling the inclusion criteria [3-6]. Then, the literature was searched in the Cochrane Back Review Group (CBRG) trial register from the last date onward for each of the interventions up to November 17, 2009.

References of relevant studies were screened and experts were approached in order to identify additional primary studies not identified in the previous steps. The language was limited to English, Dutch, French and German, because these are the languages that the authors are able to read and understand. The search strategy outlined by the CBRG was followed [7].

\section{Methods of the review}

\section{Study selection}

Two authors (NH, TK) independently screened the abstracts and titles retrieved by the search strategy and applied the inclusion criteria to all relevant abstracts. The full text version of an article was obtained if the title and abstract seemed to fulfill the inclusion criteria or if eligibility of the study was unclear. All full text articles from the existing reviews were compiled and independently screened for inclusion criteria by the authors. Any disagreements on study eligibility were resolved by discussion and a consensus meeting.

\section{Risk of bias assessment}

Two authors (NH, TK) independently assessed the risk of bias (RoB) of all eligible studies using the criteria list advised by the CBRG, which consists of 11 items [7]. Items were scored as 'positive' if they fulfilled the criteria, as 'negative' when there was a clear RoB, and as 'inconclusive' if there was insufficient information. Differences 
in assessment were discussed during a consensus meeting. A total score was computed by adding the number of positive scores, and low RoB was defined as fulfilling six or more (more than $50 \%$ ) of the 11 internal validity criteria. Empirical evidence has shown that studies fulfilling $<6$ items report higher treatment effects than studies fulfilling 6 or more items [8].

\section{Data extraction}

A standardized form was used for data extraction which included collection of descriptive data on the study population and the type of intervention, as well as quantitative data regarding the outcome measures. Data on the characteristics of the study population (gender, age), type of therapy and control treatment, adverse events and complications were also collected.

Data analysis

If studies were clinically homogeneous regarding study population, types of treatment, outcomes and measurement instruments, a meta-analysis was performed. If possible, the weighted mean difference (WMD) was calculated because this improves the interpretability of the results. If a WMD was not possible the standardized mean difference (SMD) was calculated. If trials reported outcomes as graphs, the mean scores and standard deviations (SD) were estimated from these graphs. If SD were not reported, they were calculated using the reported values of the confidence intervals, if possible. If the SD of the baseline score was reported, we used the ratio between the baseline score and $\mathrm{SD}$ to calculate the SD for other follow-up moments. Finally, if none of these data were reported, an estimation of the SD was based on study data (population and score) of other studies. In order to correct for error introduced by "double-counting" of subjects of "shared" interventions (i.e. 2 comparisons within 1 study that used the same control group as contrast) in the meta-analyses, the number of subjects in the control group was divided by the number of comparisons that this one study added in the metaanalyses. For the comparisons where studies were too heterogeneous, no meta-analysis was performed.

Quality of the evidence

Grades of recommendation, assessment, development and evaluation (GRADE) profiles were used to evaluate the overall quality of the evidence and the strength of the recommendations [9]. The quality of the evidence for a specific outcome was based upon five principal factors: (1) limitations (for example due to study design), (2) inconsistency of results, (3) indirectness (e.g. generalizability of the findings), (4) imprecision (e.g. sufficient data) and (5) other considerations, such as reporting bias. The overall quality was considered to be high when multiple RCTs with a low RoB provide consistent, generalizable, and precise data for a particular outcome. The quality of the evidence was downgraded by one level when one of the factors described above was not met [9]. Single studies were considered inconsistent and imprecise (i.e. sparse data) and provide "low quality evidence", which could be further downgraded to "very low quality evidence" if there were also limitations in design or indirectness. The following grading of quality of the evidence was applied [10]:

High quality Further research is very unlikely to change our confidence in the estimate of effect.

Moderate quality Further research is likely to have an important impact on our confidence in the estimate of effect and may change the estimate.

Low quality Further research is very likely to have an important impact on our confidence in the estimate of effect and is likely to change the estimate.

Very low quality We are very uncertain about the estimate.

\section{Results}

Description of studies

\section{Study selection}

From three existing reviews a total of 83 references were screened for eligibility. Of these 83 articles, 11 studies fulfilled the inclusion criteria and were included in this overview (Fig. 1). The most important reason for exclusion was inclusion of acute or sub-acute patients in the study or lack of information about the duration of the complaints prior to the study. Additionally, 14 potentially relevant titles and abstracts were identified in the CBRG trial register for injection-related interventions and screened for potential inclusion. All of these abstracts fulfilled the inclusion criteria and were therefore included. Two additional studies were found by forward citation searching of included studies.

In total 27 studies were included in this review. The study characteristics of all included (Appendix 1) and excluded studies (Appendix 2) are summarized in appendices. 
Fig. 1 Flow diagram of selection process of studies on injection therapy for chronic LBP

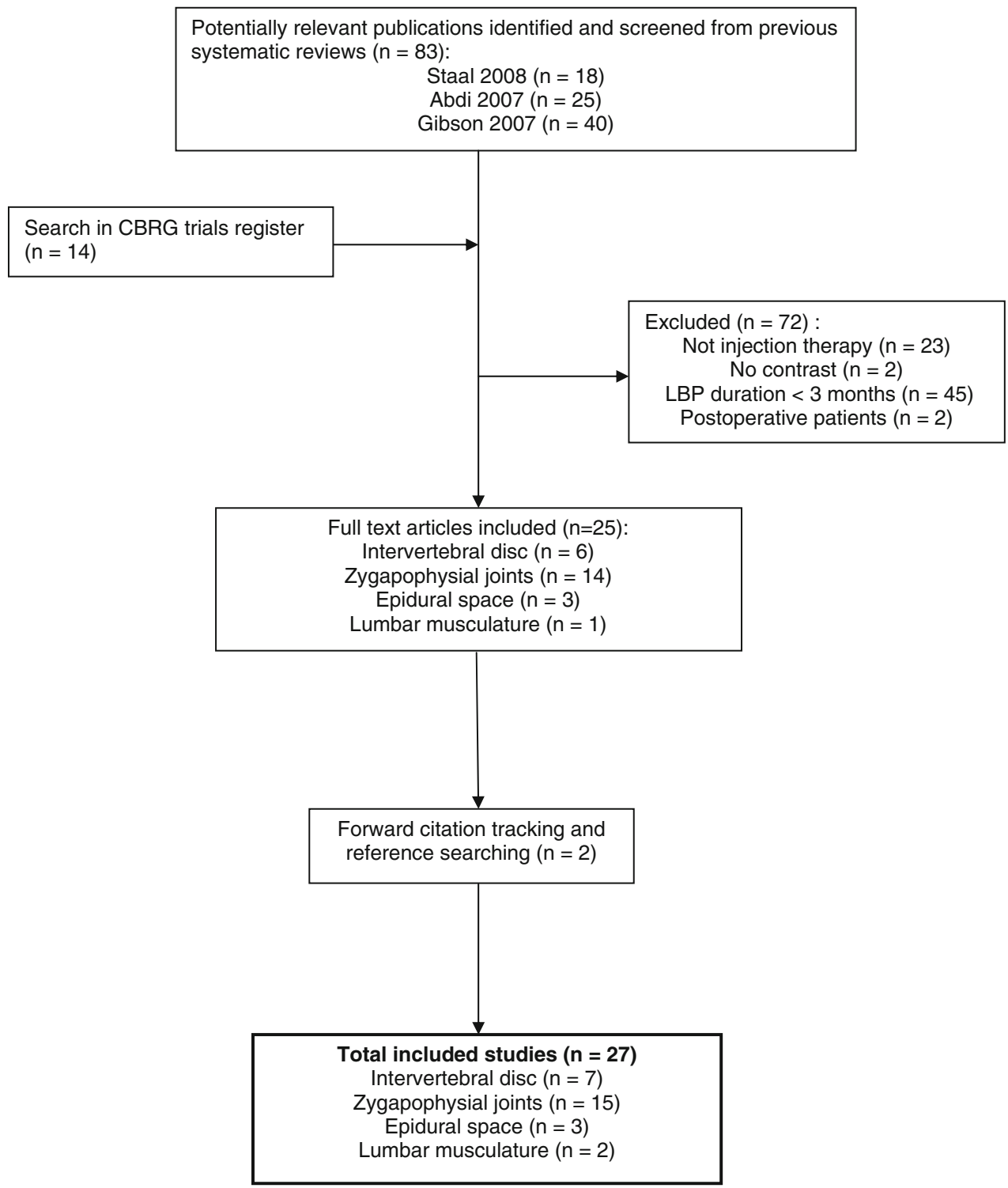

\section{Injection therapy}

Intervertebral disc One RCT was identified which evaluated injection therapy aimed at the intervertebral disc in chronic LBP patients [11]. The authors compared chemonucleolysis (intradiscal injection of chymopapain which digests the nucleus pulposus) to discectomy in patients with disc herniation which was confirmed by myelography [11]. This trial enrolled patients with both sub-acute and chronic LBP, but presented selected outcomes for the subgroup of patients $(n=58)$ with a duration of symptoms $>6$ months.

Zygapophysial joints (facet joints) Eight trials, three of which were placebo controlled [12-14], evaluated intraarticular or peri-articular facet joint injections with corticosteroids (methyl-prednisolone, triamcinolone, or betamethasone) [12, 15], local anaesthetic (lidocaine, bupivacaine, or lignocaine) [14], sodium hyaluronate [16], Sarapin (a suspension of powdered pitcher plant in alkaline solution) [17], or a combination of these [13, 18, 19]. Three of these trials confirmed that pain was originating from the facet joint by including only patients with a positive response to local anaesthetic nerve block $[12,17,18]$. One trial used radiographic confirmation of facet joint arthritis as an inclusion criterion [16] while the remaining four trials used clinical criteria (e.g. unilateral pain, pain with sustained postures) to identify patients with facet joint pain [13-15, 19].

Epidural space Three RCTs were identified that evaluated injections of corticosteroids [20, 21] or anaesthetic [22] into the epidural space. There were no placebo-controlled trials of injections into the epidural space. 
Comparisons in the included trials were made with benzodiazepine [21], spinal endoscopy [20] or between ropivacaine and bupivacaine [22]. One study excluded patients with a previous history of spinal surgery [20].

Spinal muscles (local injections) Two RCTs were identified that evaluated local intramuscular injections for nonspecific chronic LBP. One study compared injections of vitamin B12 with placebo [23], while the other compared injections of botulinum toxin A with placebo [24].

\section{Denervation procedures}

Intervertebral disc Six RCTs were identified which evaluated denervation procedures [25-30] for chronic LBP targeted at or around the intervertebral disc. Five of the trials only included patients with positive responses to either analgesic [25] or provocative [26-29] discography.

Three trials evaluated the use of percutaneous intradiscal radiofrequency thermocoagulation (PIRFT). PIRFT involves the placement of an electrode or catheter into the intervertebral disc and applying an alternating radiofrequency current to reduce nociceptive input from the disc. Two of the trials were placebo controlled [25, 28], while the other compared high- with low-intensity PIRFT [26].

Two trials were identified which compared the use of intradiscal electrothermal therapy (IDET) with a placebo [27, 29]. IDET is similar to PIRFT and involves the insertion of an electrode into the annulus or nucleus of the intervertebral disc and application of electrothermal energy to alter the pain receptors.

One RCT evaluated radiofrequency denervation of the ramus communicans nerve compared with placebo denervation in patients who failed to respond to IDET [30]. This denervation is performed outside the intervertebral disc, unlike IDET and PIRFT.

Zygapophysial joints (facet joints) Six trials compared radiofrequency denervation of the medial branch of the dorsal ramus with a sham denervation procedure [31-36], and one trial compared radiofrequency denervation of the dorsal root ganglion with a sham procedure [37]. The trial by Tekin and colleagues [34] included comparisons between conventional, pulsed, and sham radiofrequency denervation. All trials except one [32] included only patients with a positive response ( $\sim 50-80 \%$ pain relief) to selective anaesthetic nerve blocks. The trial by Leclaire and colleagues [32] included patients who experienced significant relief of their LBP for at least $24 \mathrm{~h}$ during the week after intra-articular facet injections under fluoroscopy. This inclusion criterion was considered to be insufficiently sensitive and likely to have included patients with pain of non-facet joint origin [32]. Therefore, this trial was not included in the primary analysis, but a sensitivity analysis was performed to evaluate the addition of this study to the meta-analyses.

Risk of bias in included studies

The results of the RoB assessment are shown in Table 1. 18 studies $(66 \%)$ had a low RoB. All studies were described as randomized; however, only nine studies (33\%) used an adequate randomization procedure in combination with an adequate concealment of treatment allocation. In only four studies $(15 \%)$ co-interventions were avoided or similar between groups. Many studies had acceptable compliance (20 studies; 77\%) or acceptable drop-out rates (21 studies; $77 \%$ ) or both (15 studies; $58 \%$ ).

Effects of intervention

\section{Feasibility of statistical pooling}

As stated in the methods section, statistical pooling was only considered if subgroups of studies were clinically homogeneous, and the authors provided sufficient information on study characteristics, outcome measures, and study results. After reviewing the included study characteristics, only two treatment subgroups (IDET vs. placebo and facet joint denervation vs. placebo) were sufficiently clinically homogeneous to perform statistical pooling (Table 2).

Injection therapy

\section{Intervertebral disc}

Chemonucleolysis versus other treatment In one RCT with a high RoB, chemonucleolysis (via intradiscal chymopapain injection) was compared with discectomy [11]. A subgroup of 68 patients had duration of symptoms $>6$ months prior to the start of the trial. There was no difference between groups in percentage of patients whose pain had disappeared or improved (87 vs. 85\%) 12 months post-treatment. Within 12 months post-treatment, $25 \%$ of the chemonucleolysis group were considered treatment failures and underwent surgery.

There is very low quality evidence ( $1 \mathrm{RCT} ; n=68$; limitations in design, inconsistency, imprecision) that chemonucleolysis is no more effective than discectomy over a long-term follow-up.

\section{Zygapophyseal joint (facet joint)}

Facet joint injections with corticosteroids versus placebo Two RCTs, one with low RoB [12] and one with high RoB [13], compared the effects of facet joint injections with 


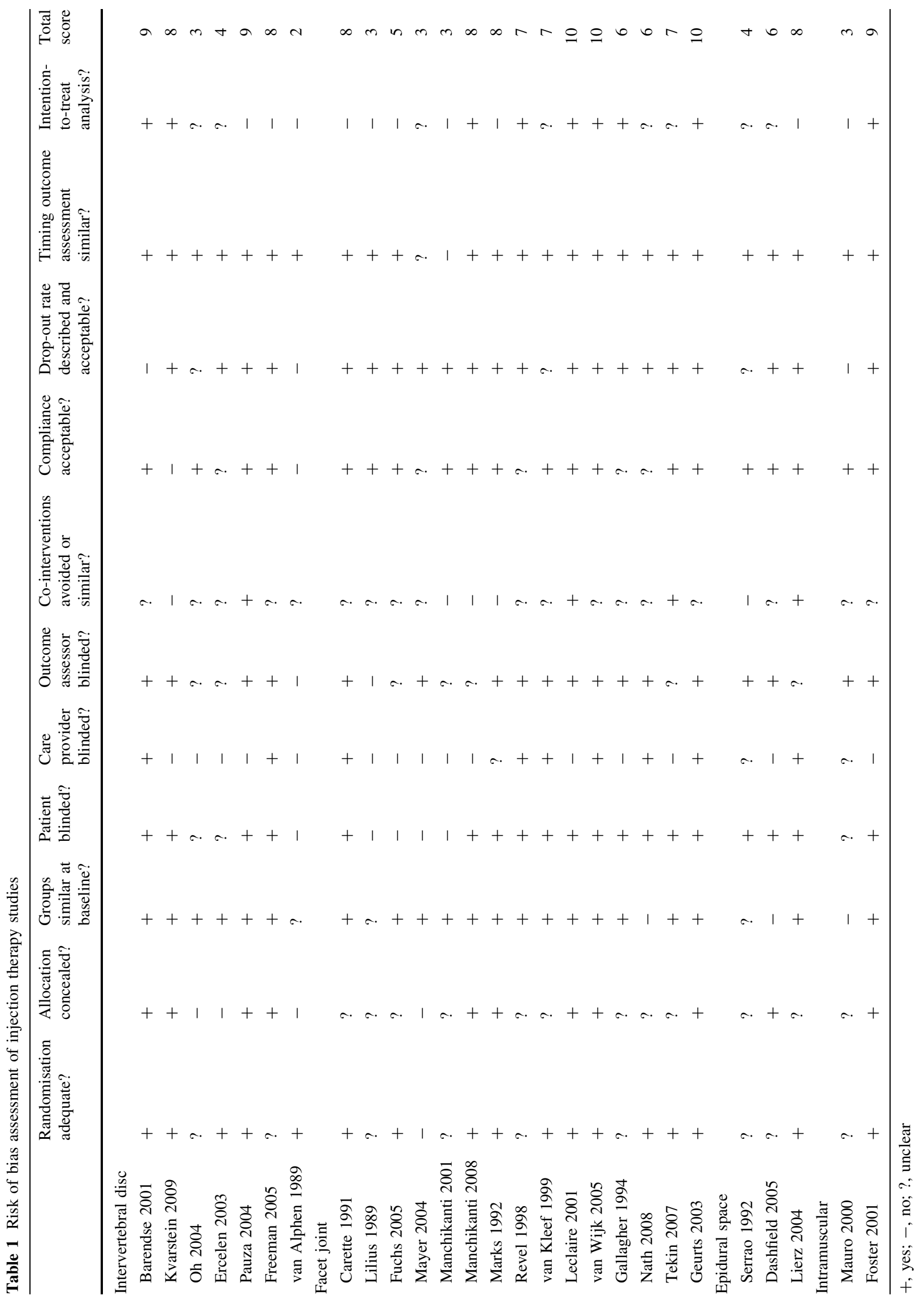


Table 2 Pooled effect estimates for injection therapy and denervation procedures

\begin{tabular}{|c|c|c|c|c|c|}
\hline \multicolumn{2}{|c|}{ Outcome title } & $\begin{array}{l}\text { No. of } \\
\text { studies }\end{array}$ & $\begin{array}{l}\text { No. of } \\
\text { participants }\end{array}$ & Statistical method & Effect size \\
\hline \multicolumn{6}{|c|}{ Comparison 01: IDET versus placebo for chronic non-specific LBP } \\
\hline 01 & $\begin{array}{l}\text { Change in pain intensity from baseline } \\
\text { on SF-36 Bodily Pain Index (100-point). } \\
\text { Long-term follow-up ( } 6 \text { months) }\end{array}$ & 2 & 111 & $\begin{array}{l}\text { Weighted mean difference } \\
\text { (fixed) } 95 \% \mathrm{CI}\end{array}$ & $-7.84[-14.96,-0.72]$ \\
\hline 02 & $\begin{array}{l}\text { Change in functional status from baseline } \\
\text { on Oswestry Disability Index (100-point). } \\
\text { Long-term follow-up (6 months) }\end{array}$ & 2 & 111 & $\begin{array}{l}\text { Weighted mean difference } \\
\text { (fixed) } 95 \% \mathrm{CI}\end{array}$ & $-4.93[-10.11,0.25]$ \\
\hline \multicolumn{6}{|c|}{ Comparison 02: radiofrequency denervation of lumbar facet joints compared with placebo for chronic LBP } \\
\hline 01 & $\begin{array}{l}\text { Change in pain intensity from baseline } \\
\text { on 100-point VAS. Short-term } \\
\text { follow-up ( } \leq 4 \text { weeks) }\end{array}$ & 2 & 90 & $\begin{array}{l}\text { Weighted mean difference } \\
\text { (fixed) } 95 \% \text { CI }\end{array}$ & $-18.15[-24.21,-12.09]$ \\
\hline 02 & $\begin{array}{l}\text { Change in pain intensity from baseline } \\
\text { on 100-point VAS. Intermediate-term } \\
\text { follow-up (1-6 months) }\end{array}$ & 2 & 112 & $\begin{array}{l}\text { Weighted mean difference } \\
\text { (random) } 95 \% \text { CI }\end{array}$ & $-9.29[-22.57,4.00]$ \\
\hline 03 & $\begin{array}{l}\text { Change in pain intensity from baseline } \\
\text { on 100-point VAS. Long-term } \\
\text { follow-up ( } 6 \text { months) }\end{array}$ & 3 & 130 & $\begin{array}{l}\text { Weighted mean difference } \\
\text { (random) } 95 \% \text { CI }\end{array}$ & $-6.99[-14.73,0.76]$ \\
\hline 04 & $\begin{array}{l}\text { Change in functional status from baseline } \\
\text { on Oswestry Disability Index. } \\
\text { Short-term follow-up ( } \leq 4 \text { weeks) }\end{array}$ & 1 & 60 & $\begin{array}{l}\text { Weighted mean difference } \\
\text { (fixed) } 95 \% \text { CI }\end{array}$ & $-5.53[-8.66,-2.40]$ \\
\hline
\end{tabular}

For forest plots and GRADE evidence profiles, see Appendix 3

corticosteroids to placebo injections. There was insufficient data on pain and functional status in the Lilius study [13] to allow for statistical pooling of outcomes. In the Carette study [12], no significant differences were found between the groups at 1 and 3 months for pain, functional status or selfrated improvement. At the 6-month follow-up, significant differences were found in favour of the corticosteroid group [12]. The high RoB study [13] compared intra-articular and peri-capsular corticosteroid injections with placebo injections. No significant differences between the groups were reported for pain, disability or work attendance at either short- or intermediate-term follow-ups [13]. No side effects apart from transient pain were reported.

There is very low quality evidence ( $1 \mathrm{RCT} ; n=97$; inconsistency, indirectness, imprecision) that there is no significant difference in effect between facet joint injections with corticosteroids and placebo injections for short- to intermediate-term pain relief and improvement of function.

Facet joint injections with corticosteroids versus other treatment Five RCTs compared the effects of corticosteroids injections into and around the facet joints with other treatments [15-19]. Because of the clinical heterogeneity of the reference treatments, pooling was determined to be unsuitable.

In a study with low RoB [19], intra-articular facet joint injections with corticosteroids and lignocaine were compared with facet nerve blocks using similar medication. The facet joint injections provided slightly better pain relief than facet nerve blocks, although statistical significance was only reached at 1 month, not immediately post-treatment or after 3 months [19].

Two RCTs with high RoB compared intra-articular facet joint corticosteroid injections with other treatments; one compared facet joint injections with a mixture of local anaesthetics and corticosteroids combined with a home stretching exercise program with the home stretching exercise program only [15]. No significant post-treatment differences between the groups were found for pain and disability. The other trial compared the effects of facet joint corticosteroid injections with intra-articular sodium hyaluronate injections. No significant differences in pain relief, disability and quality of life between the groups were found at different follow-up points over a 6-month period [16].

One RCT with low RoB [18] compared the effects of multiple medial branch blocks of corticosteroids combined with local anaesthetics with multiple medial branch blocks consisting of only local anaesthetics. No significant differences between the groups were found at 3,6 or 12 months post-treatment. One RCT with high RoB [17] compared the effects of multiple medial branch blocks of corticosteroids combined with local anaesthetics and Sarapin with multiple medial branch blocks consisting of local anaesthetics and Sarapin. No significant differences between the groups were found for pain relief, overall health, functional status and return-to-work over more than 2 years of follow-up.

There is low quality evidence ( $1 \mathrm{RCT} ; n=86$; inconsistency, imprecision) that intra-articular facet joint 
injections are slightly more effective than facet nerve blocks for pain relief in the intermediate term. There is very low quality evidence ( $1 \mathrm{RCT} ; n=70$; limitations in design, inconsistency, imprecision) that intra-articular facet joint injections add no benefit to a home exercise stretching program in terms of pain and disability over the short term. There is very low quality evidence ( $1 \mathrm{RCT} ; n=60$; limitations in design, inconsistency, imprecision) that there is no significant difference in effect between intra-articular facet joint corticosteroid injections and intra-articular injections of sodium hyaluronate on pain and disability over a long-term follow up. There is very low quality evidence ( $1 \mathrm{RCT} ; n=120$; inconsistency, indirectness, imprecision) that the addition of corticosteroids does not increase the effectiveness of facet joint nerve blocks with local anaesthetic. There is very low quality evidence (1 RCT; $n=73$; limitations in design, inconsistency, indirectness, imprecision) that the addition of corticosteroids does not increase the effectiveness of facet joint nerve blocks with anaesthetic and Sarapin in chronic LBP.

Facet joint injections with local anaesthetic versus placebo One RCT with low RoB [14] compared intraarticular facet joint injections with lidocaine to intraarticular facet joint injections with saline. In both groups these injections were followed by an injection of corticosteroid (cortivazol) near the joints. The lidocaine group had significantly higher pain relief post-treatment than the saline group [14].

There is low quality evidence ( $1 \mathrm{RCT} ; n=80$; inconsistency, imprecision) that intra-articular facet joint injections with lidocaine combined with peri-articular corticosteroid injections are more effective for short-term pain relief than placebo.

\section{Epidural space}

Epidural corticosteroid injections versus other treatments In an RCT with high RoB [21], an epidural injection with a corticosteroid and dextrose solution was compared with an intrathecal benzodiazepine with dextrose injection. Two weeks and 2 months post-treatment, no significant differences between the groups were reported for pain relief or general improvement.

One RCT with low RoB [20] compared caudal epidural local anaesthetic and steroid injection with targeted epidural local anaesthetic and steroid placement with a spinal endoscope. No significant differences were found between the groups for any of the outcome measures at any of the times. In all patients in the endoscope group, post-treatment lowback discomfort was experienced but this was not persistent.

There is very low quality evidence ( 2 RCTs; $n=88$; limitations in design, imprecision, inconsistency) that epidural corticosteroid injection is not significantly different to benzodiazepine injection or targeted epidural placement for pain relief over the short to intermediate term.

Epidural injections with local anaesthetics versus other treatments One RCT with low RoB [22] compared the effects of epidural blocks with ropivacaine with epidural blocks with bupivacaine. Eight single shot epidural injections followed by active physiotherapy were performed in all patients. There were no significant differences found between the groups in post-treatment analgesia. There were three cases of short episodes of headache post-injection.

There is low quality evidence ( $1 \mathrm{RCT} ; n=40$; imprecision, inconsistency) that there is no significant difference in analgesia provided by blocks of ropivacaine or bupivacaine in the short term.

\section{Lumbar musculature}

Intramuscular injections with vitamin $B 12$ versus placebo In one RCT with high RoB [23], the effects of intramuscular vitamin B12 injections were compared with intramuscular placebo injections. Post-treatment, there were significant improvements for pain and disability in favour of the vitamin B12 group.

There is very low quality evidence ( $1 \mathrm{RCT} ; n=60$; limitations in design, inconsistency, imprecision) that intramuscular vitamin B12 injections are more effective than intramuscular placebo injections for short-term pain relief and improvement of function.

Intramuscular injections with botulinum toxin A versus placebo In one RCT with low RoB, intramuscular injections of botulinum toxin A were compared with intramuscular placebo injections of saline [24]. At 3 weeks follow-up, the degree of pain relief was significantly different between groups in favour of the botulinum toxin A group. At 8 weeks patients in the botulinum toxin A group had significantly more pain relief and better ODI scores than the placebo group.

There is low quality evidence ( $1 \mathrm{RCT} ; n=31$; inconsistency, imprecision) that intramuscular botulinum toxin A injections are more effective for pain relief in the shortand intermediate-term than placebo.

Denervation procedures

\section{Intervertebral disc}

Percutaneous intradiscal radiofrequency thermocoagulation versus placebo In one placebo-controlled trial $(n=28)$ with low RoB, no significant differences were found between PIRFT and sham PIRFT in pain VAS 
scores, global perceived effect, ODI, or a composite outcome of overall treatment success 8 weeks post-treatment [25]. In a second placebo-controlled trial $(n=20)$ with low RoB, only follow-up data which were collected after 6 and 12 months post-treatment were reported [28]. No significant differences were seen between the PIRFT and sham-PIRFT groups on pain intensity or functional status at either of these time points. Because of the variability in the timing of outcome measures between these two studies, a decision was made not to pool the results.

A third trial $(n=37)$ with high RoB found minimal improvement over 6 months on pain (VAS) and disability (ODI) with both lower- and higher- intensity of PIRFT. No significant differences were found between the groups at any of the follow-up assessments [26]. No complications or adverse events were reported in the placebo-controlled trials. One patient was excluded from the analysis of Ercelen et al. [26] because of discitis.

There is low quality evidence ( $1 \mathrm{RCT} ; n=28$; inconsistency; imprecision) that there is no difference between PIRFT and placebo in pain and functional status outcomes over an intermediate term of follow-up. There is also low quality evidence ( $1 \mathrm{RCT} ; n=20$; inconsistency; imprecision) that there is no difference between PIRFT and placebo in pain and functional status outcomes over a longterm follow-up.

Intradiscal electrothermal therapy versus placebo: pain In patients with a positive response to provocative discography, two small ( $n=55$ and $n=56$ ), low RoB, placebo-controlled randomized trials evaluated IDET and both provided sufficient data for pooling [27, 29]. Both studies measured pain with the SF-36 Bodily Pain Index (100point scale). The Chi-square value for homogeneity of the WMD was $0.09(P>0.05)$, indicating statistical homogeneity among these studies.

There is low quality evidence ( 2 RCTs; $n=111$; indirectness, imprecision) that IDET is more effective than placebo for pain relief over a long-term (6 months) followup (WMD -7.84; 95\% CI -14.96 to -0.72) (Table 2, Analysis 01.01).

Intradiscal electrothermal therapy versus placebo: functional status The same two RCTs [27, 29] provided ODI scores on a 100-point scale which allowed statistical pooling. The Chi-square value for homogeneity of the WMD was $1.37(P>0.05)$, indicating statistical homogeneity among these studies.

There is low quality evidence ( 2 RCTs; $n=111$; indirectness, imprecision) that IDET is no more effective than placebo in improving functional status over a long-term (6 months) follow-up (WMD -4.93 ; 95\% CI -10.11 to 0.25 ) (Table 2, Analysis 01.02).
In patients unresponsive to treatment with IDET, one high RoB RCT found radiofrequency denervation of the ramus communicans nerve was associated with better VAS pain, SF-36 bodily pain and SF-36 physical function scores after 4 months compared to sham denervation [30]. In one RCT, four patients who underwent IDET experienced transient radiculopathy ( $<6$ weeks) [27]. No other serious adverse events were reported in the three trials.

\section{Zygapophyseal joint (facet joint)}

Radiofrequency denervation of facet joints versus placebo: pain Five RCTs provided sufficient data on pain VAS scores to allow for pooling over a short-, intermediate- or long-term follow-up [31, 33-36]. All studies included only patients with a positive response ( $\sim 50-80 \%$ pain relief $)$ to local anaesthetic nerve block. One RCT was not included in the primary analyses due to clinical heterogeneity of patient selection procedures [32].

For short-term outcomes ( $<4$ weeks), the Chi-square value for homogeneity of the WMD was $1.07(P>0.05)$, indicating statistical homogeneity between two of the RCTs. There is low quality evidence ( 2 RCTs; $n=90$; indirectness, imprecision) that radiofrequency denervation of lumbar facet joints is more effective than placebo for pain relief over a short-term follow-up (WMD -18.15; 95\% CI -24.21 to -12.09 ) (Table 2, Analysis 02.01).

For intermediate-term outcomes (1-6 months), the Chisquare value for homogeneity of the WMD was 1.75 $(P>0.05)$. There is low quality evidence (2 RCTs; $n=112$; indirectness, imprecision) that radiofrequency denervation of lumbar facet joints is no more effective than placebo for pain relief in the intermediate term (WMD -9.29; 95\% CI -22.57 to 4.00) (Table 2, Analysis 02.02).

For long-term outcomes (6 months), the Chi-square value for homogeneity of the WMD was $4.58(P>0.05)$. There is low quality evidence ( 3 RCTs; $n=130$; indirectness, imprecision) that radiofrequency denervation of lumbar facet joints is no more effective than placebo for pain relief in the long term (WMD -6.99; 95\% CI - 14.73 to 0.76) (Table 2, Analysis 02.03).

When the study by Leclaire and colleagues [32] was included in the analyses, the pooled WMD (95\% CI) for pain intensity was $-14.80(-22.77$ to -6.82$)$ in the short-term and $-3.85(-16.27$ to 8.57$)$ in the intermediate term. While the addition of this study to the meta-analyses slightly altered the pooled WMD, it did not change the conclusions.

One RCT with low RoB [37] compared radiofrequency denervation of the dorsal root ganglion with sham denervation. No significant differences were found between groups at 3-month follow-up. Adverse events and complications did not differ between treatments, and no serious complications or side effects arose in either group. There is 
very low quality evidence ( $1 \mathrm{RCT} ; n=83$; inconsistency, indirectness, imprecision) that radiofrequency denervation of the dorsal root ganglion is no more effective than placebo for pain relief in the intermediate term.

Radiofrequency denervation of facet joints versus placebo: functional status One RCT comparing conventional and pulsed radiofrequency denervation to placebo provided sufficient data on functional status outcomes (ODI 0-100 scale) to allow for statistical pooling [34].

There is very low quality evidence ( $1 \mathrm{RCT} ; n=60$; inconsistency, indirectness, imprecision) that radiofrequency denervation of lumbar facet joints is more effective than placebo for improvement of function in the short term (WMD $-5.53 ; 95 \%$ CI -8.66 to -2.40 ) (Table 2, Analysis 02.04).

When the study by Leclaire and colleagues [32] was included in the analyses, the WMD (95\% CI) became $-3.45(-7.68$ to 0.77$)$ in the short term and $-6.57(-17.00$ to 3.85 ) in the intermediate term (when pooled with the study by van Kleef et al. [35]). While the addition of this study to the meta-analyses slightly altered the short-term pooled WMD, it also changed the GRADE profile and the overall quality of the evidence from very low to low.

\section{Radiofrequency denervation of facet joints versus other} treatment In a study with low RoB [34], conventional radiofrequency denervation of the lumbar facet joints was compared with pulsed radiofrequency denervation. Both treatments improved pain VAS and ODI scores compared with placebo, with conventional denervation improving significantly more than pulsed denervation by 6 months and 1 year post-treatment.

There is very low quality evidence ( $1 \mathrm{RCT} ; n=40$; inconsistency, indirectness, imprecision) that conventional radiofrequency denervation is more effective than pulsed radiofrequency denervation of the facet joints for pain relief or improvement of function over the long term.

\section{Discussion}

In this review, 27 RCTs were included that evaluated the effectiveness of injection therapy and denervation procedures for chronic LBP.

The effectiveness of the different therapies

In this review we found only low to very low quality evidence to support the use of injection therapy and denervation procedures in chronic LBP patients. Low quality evidence means that further research is very likely to have an important impact on our confidence in the estimate of effect and is likely to change the estimate.
From the 26 included RCTs, only two treatment subgroups (IDET vs. placebo, and radiofrequency denervation of facet joints vs. placebo) were considered to be clinically homogeneous enough to allow for statistical pooling. The results showed that IDET is more effective than placebo for pain relief over 6 months of follow-up and radiofrequency denervation of lumbar facet joints more effective than placebo for pain relief over a short-term $(<4$ weeks) follow-up. The quality of the evidence in both of these comparisons was downgraded to low quality due to imprecision and indirectness. Indirectness (lack of ability to generalize) refers to the extent to which the people, interventions and outcomes in the trials are not comparable to those defined in the inclusion criteria of the review. As all of the pooled RCTs selected patients for inclusion only if they had a positive response to either provocative discography (IDET vs. placebo) or local anesthetic nerve block (radiofrequency denervation of facet joints vs. placebo). While the selection of appropriate patients may be considered necessary for these therapies, the results cannot be generalized to all patients with chronic LBP. However, from the perspective of pain clinics or anesthesiologists these populations may be generalizable to their setting. In that case, the level of evidence is moderate, indicating that further research is likely to have an important impact on our confidence in the estimate of effect and may change the estimate. Consequently, further research is needed for a more confident estimate of effect.

\section{Methodological considerations}

The RoB assessment describes whether a RCT reported certain methodological features known to decrease bias when interpreting the results. However, despite the fact that the RoB of the included studies was generally low, many studies showed flaws regarding concealment of treatment allocation, care provider blinding, avoidance of co-interventions and performing intention-to-treat analyses. Overall, only low to very low quality evidence was found by this review. By using the GRADE approach, we were able to determine an overall judgment of the quality of the evidence not only from the limitations in design, but also considering aspects such as inconsistency among studies, imprecision and indirectness.

It is recommended that study features which are associated with a lower RoB in RCTs be considered when designing future trials of non-surgical interventional therapy, as well as adequate reporting of these features.

Adverse effects

In the majority of studies presented in this review, no adverse events or side effects associated with the 
treatments were reported. Transient symptoms such as increased low-back discomfort or paraesthesia were noted in a few studies. Epidural injections were associated with nausea and headache in some patients. In a study which used discectomy as a reference treatment [11], a dural defect with leakage of cerebrospinal fluid and a partial cauda equina were noted, though unsure whether these patients were in the injection therapy or surgery group. Most trials were small and not designed to evaluate adverse events, so no clear conclusion can be drawn regarding the risks of injection therapy and denervation procedures.

\section{Strengths and limitations}

Several biases can be introduced in systematic reviews by literature search and selection procedures. It is possible that in searching studies for this review, relevant but unpublished trials may have been missed, which are often likely to be small studies without positive results, leading to publication bias. However, because the majority of published trials was small and did not show a positive effect, publication bias does not seem to be a big problem in this review. Screening references of identified trials and systematic reviews may result in an over representation of positive studies in the review, because trials with a positive result are more likely to be referred to in other publications, leading to reference bias. Only studies published in English, Dutch, French or German were included in this review. It is not clear whether a language restriction is associated with bias [38].

Due to the study selection criterion used, including only trials of chronic ( $>3$ months duration) LBP patients, this review included less studies than similar Cochrane reviews $[3,5]$. Many excluded RCTs failed to explicitly state the duration of symptoms experienced by the patients, while others considered only the length of time patients were unresponsive to conservative treatment as part of their inclusion criteria. However, the main conclusions of our review appear to be similar to recent systematic reviews [3-6], which generally report a paucity of RCTs on injection therapy and denervation procedures for chronic LBP and insufficient evidence to support their use.

Implications for research

To conclude, we identified 27 RCTs that evaluated various types of injection therapy or denervation procedures at different locations for patients with chronic LBP. Most of the studies included in this review were small and had a low RoB, though there were methodological weaknesses, especially regarding concealment of allocation, co-interventions and use of intention-to-treat analyses. The quality of evidence was low or very low, indicating that further research is very likely to have an important impact on our confidence in the estimate of effect and is likely to change the estimate. There is a need for future high-quality placebo-controlled RCTs with large sample sizes on injection therapy and denervation procedures.

Implications for practice

In patients with chronic LBP there is only low to very low quality evidence to support the use of injection therapy for pain relief and improvement of function. It cannot be ruled out that in carefully selected sub-groups of patients, such as those with a positive response to discography or local anaesthetic nerve block, certain interventional therapies may be of some benefit. Potential benefits must be weighed against possible adverse effects when deciding whether to provide injection therapy or denervation to chronic LBP patients.

Open Access This article is distributed under the terms of the Creative Commons Attribution Noncommercial License which permits any noncommercial use, distribution, and reproduction in any medium, provided the original author(s) and source are credited.

\section{Appendix 1}

See Table 3.

Table 3 Characteristics of included studies

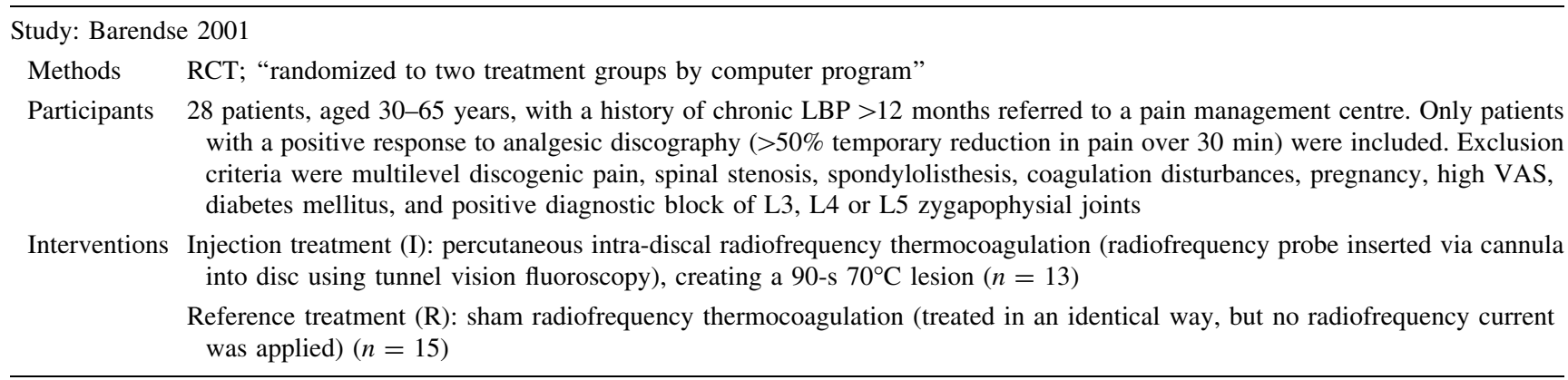


Table 3 continued

Outcomes No significant differences were found for any of the primary or secondary outcomes after 8 weeks post-treatment. Change in VAS was -0.61 in (I) and -1.14 in (R); while change in Oswestry scale was -2.62 in (I) and -4.93 in (R)

Notes $\quad$ No complications during or after the procedures were reported.

Methodological quality: randomization + ; treatment allocation + ; baseline similarity + ; patients blinded + ; care providers blinded +; outcomes blinded +; co-interventions ?; compliance + ; drop outs - ; timing of outcomes + ; intention-to-treat + . Total score $=9$

Study: Carette 1991

Methods RCT; "generated from a table of random numbers"

Participants 101 patients, aged 18-65 years, with chronic LBP and a positive response to lumbar facet joint anaesthetic block. Exclusion criteria included specific LBP, previous injections into the facet joints, previous low-back surgery, pregnancy, known allergy to local anaesthetic and the presence of a blood coagulation disorder

Four patients were later excluded from the analysis.

Interventions Injection treatment (I): lumbar facet joint injection of corticosteroid (1 ml methylprednisolone acetate mixed with $1 \mathrm{ml}$ isotonic saline) $(n=49)$

Reference treatment (R): sham lumbar facet joint injection $(2 \mathrm{ml}$ isotonic saline) $(n=48)$

Outcomes One to 3 months post-treatment, the two groups did not differ clinically or statistically in any of the outcome measures. By 6 months post-treatment, patients treated with (I) reported significantly better VAS (mean difference $-1.0,95 \% \mathrm{CI}-2.0$ to 0.1 ) and sickness impact profile-physical dimension scores (mean difference $-3.0,95 \% \mathrm{CI}-5.5$ to -0.5 ) compared with (R)

Notes No adverse events were reported, other than transient local pain at the injection sites.

Methodological quality: randomization + ; treatment allocation ?; baseline similarity + ; patients blinded + ; care providers blinded +; outcomes blinded +; co-interventions ?; compliance +; drop outs +; timing of outcomes +; intention-to-treat - . Total score $=8$

Study: Dashfield 2005

Methods RCT; "allocated randomly"

Participants 60 patients, aged 18 years or more, with sciatica (defined as pain in the distribution of a lumbar nerve root, \pm neurosensory and motor deficits) for a minimum of 6 months, but not for longer than 18 months. Patients with previous spinal surgery, coagulopathy, progressive motor neurone disorders, or peripheral vascular disease were excluded

Interventions Injection treatment (I): caudal epidural corticosteroid injection (10 ml lidocaine $1 \%$ with $40 \mathrm{mg}$ triamcinolone) injected into the epidural space $(n=33)$

Injection treatment (I2): lumbar epiduroscopy (painful nerve root identified and instilled with $10 \mathrm{ml}$ lidocaine $1 \%$ with triamcinolone $40 \mathrm{mg})(n=27)$

Outcomes No significant differences were found between the groups after 6 weeks, 3 months and 6 months post-treatment for any of the measures. For the (I) group, there were more significant differences between pre-treatment and post-treatment outcome measures compared with the (I2) group

Notes Non-persistent post-procedure low-back discomfort occurred in all (I2) patients, and in fewer (I) patients

Methodological quality: randomization ?; treatment allocation + ; baseline similarity - ; patients blinded + ; care providers blinded -; outcomes blinded +; co-interventions ?; compliance +; drop outs +; timing of outcomes + ; intention-to-treat ? Total score $=6$

Study: Ercelen 2003

Methods RCT; "patients were randomized into two treatment groups by computer"

Participants 39 patients with chronic LBP who had been receiving conservative treatment for at least 2 years. Patients with spinal stenosis, instability, spondylolisthesis, diabetes mellitus, tumour infiltration, coagulation disorders, clinical radiculopathy, other neurologic abnormalities or systemic inflammatory diseases were excluded from the study. Only patients with a positive provocative discography at L4-L5, L5-S1, or both locations were randomized

Two patients were excluded from the analysis (one with discitis, one lost to follow up)

Interventions Injection treatment (I1): percutaneous intradiscal radiofrequency thermocoagulation (radiofrequency probe inserted via cannula into disc using fluoroscopy) at $80^{\circ} \mathrm{C}$. Lesioning was performed for $120 \mathrm{~s}(n=19)$

Injection treatment (I2): percutaneous intradiscal radiofrequency thermocoagulation, identical procedure to (I1) but lesioning was performed at $80^{\circ} \mathrm{C}$ for $360 \mathrm{~s}(n=18)$

Outcomes No significant differences were observed between groups at any of the follow-up time points (post-treatment, 1 and 2 weeks, 1 , 3 , and 6 months). Post-treatment to 1 month scores on pain VAS and Oswestry disability scale decreased significantly compared with pre-treatment scores $(P<0.05)$. There were no statistical differences between the final $(6$ month $)$ and the pretreatment VAS and ODS values in both groups 
Table 3 continued

Notes $\quad$ Adverse events were not reported

Methodological quality: randomization +; treatment allocation -; baseline similarity +; patients blinded ?; care providers blinded -; outcomes blinded ?; co-interventions ?; compliance ?; drop outs +; timing of outcomes +; intention-to-treat ?. Total score $=4$

Study: Foster 2001

Methods RCT; "randomly assigned by drawing a card from a deck of shuffled cards"

Participants 31 patients aged $>18$ years with chronic LBP greater on one side than the other. Exclusion criteria were LBP for $<6$ months, presence of a systemic inflammatory disorder, acute pathology on MRI, known allergy or sensitivity to botulinum toxin, pregnancy, disorders of neuromuscular transmission and anaesthetic or corticosteroid injections to the lumbar spine within 12 weeks of enrolment

Interventions Injection treatment (I): intramuscular injections of botulinum toxin A at 5 lumbosacral sites, with each site receiving 40 units $(n=15)$

Reference treatment (R): intramuscular injections of saline to at 5 lumbosacral sites, with each site receiving 40 units $(n=16)$

Outcomes At 3 weeks, the degree of pain relief exceeded 50\% (VAS score) in 11 of 15 patients (73.3\%) in the (I) group compared with four of $16(25 \%)$ in the $(\mathrm{R})$ group $(P<0.012)$. The difference between these groups was $48 \%$ (95\% CI 11.7-80.1). At 8 weeks, 9 of 15 patients $(60 \%)$ in the (I) group and 2 of $16(12.5 \%)$ in the (R) group reported pain relief exceeding $50 \%$ $(P<0.009)$, a difference of $47.5 \%$ (95\% CI 10.5-79.1). For the Oswestry, 10 of 15 patients $(66.7 \%)$ in the (I) group and 3 of $14(18.8 \%)$ in the $(\mathrm{R})$ group demonstrated improvement at 8 weeks $(P<0.011)$. The difference between groups was $47.9 \%$ (95\% CI 10.9-79.6)

Notes Injections were well tolerated by all patients and none had side effects

Methodological quality: randomization + ; treatment allocation + ; baseline similarity + ; patients blinded + ; care providers blinded -; outcomes blinded + ; co-interventions ?; compliance + ; drop outs + ; timing of outcomes + ; intention-to-treat + . Total score $=9$

Study: Freeman 2005

Methods RCT; "randomization schedule"

Participants 57 patients with chronic LBP and evidence of degenerative disc disease on MRI. All subjects had one- or two-level symptomatic disc degeneration as determined by provocative lumbar discography. Exclusion criteria included the presence of a large contained or sequestered herniation, spinal stenosis, previous back surgery, spondylolisthesis, psychological disorders, and pregnancy

Two subjects violated protocol and were not included in the analysis

Interventions Injection treatment (I): intradiscal electrothermal therapy (IDET) at $90^{\circ} \mathrm{C}$ for 4 min, followed by a rehabilitation program $(n=38)$

Reference treatment (R): sham IDET, same procedure as (I) without connection to the generator, followed by a rehabilitation program $(n=19)$

Outcomes 6 months post-treatment, there were no significant differences between groups on any of the primary or secondary outcome measures. Mean difference $(95 \% \mathrm{CI})$ between groups on the low-back outcome score was -1.71 ( -3.82 to 0.41$)$ and on Oswestry was -2.16 ( -8.37 to 4.86$)$ in favour of (I)

Notes There were no serious adverse events in either arm of the study

Methodological quality: randomization ?; treatment allocation + ; baseline similarity + ; patients blinded + ; care providers blinded +; outcomes blinded +; co-interventions ?; compliance +; drop outs +; timing of outcomes + ; intention-to-treat - . Total score $=8$

Study: Fuchs 2005

Methods RCT; block randomization generated by computer

Participants 60 patients with chronic LBP with radiologic confirmation of facet joint osteoarthritis. Patients with a history of hypersensitivity or contraindication to the test products, contraindication to intra-articular treatment, a current regimen of anticoagulants or radicular pain were excluded from the study

Interventions Injection treatment (I): lumbar facet joint sodium hyaluronate injections (10 mg sodium hyaluronate in $1 \mathrm{ml}$ buffer solution) bilaterally, under CT guidance at weekly intervals, for 3 weeks $(n=30)$

Injection treatment (I2): lumbar facet joint corticosteroid injection (10 $\mathrm{mg}$ triamcinolone acetonide in $1 \mathrm{ml}$ crystalline suspension) bilaterally, under CT guidance at weekly intervals, for 3 weeks $(n=30)$

Outcomes No statistically significant differences between the groups in any outcomes were reported post-treatment, after 3 and 6 months. Pain VAS in the (I) group decreased from $69.2 \pm 14.2$ to $38.0 \pm 26.5 \mathrm{~mm}$ by 3 months post-treatment. In the (I2) group the pain intensity decreased from $68.7 \pm 11.5$ to $33.4 \pm 20.7 \mathrm{~mm}$ 
Table 3 continued

Notes $\quad$ No adverse effects were reported after administration of both therapies

Methodological quality: randomization + ; treatment allocation ?; baseline similarity + ; patients blinded - ; care providers

blinded -; outcomes blinded ?; co-interventions ?; compliance +; drop outs +; timing of outcomes +; intention-to-treat -. Total score $=5$

Study: Gallagher 1994

Methods RCT; randomisation method not stated

Participants 41 patients, aged 25-55 years, with chronic LBP who fulfilled criteria for facet joint pain (including tenderness on palpation; pain on extension or rotation; pain exacerbated by exercise, sitting, and standing; and radiological evidence of facet joint degeneration). Subjects had a positive response to local anaesthetic injection into and around the painful joint. Exclusion criteria included previous back surgery, signs of nerve root compression, major mental illness, pending compensation claims and general ill health

Interventions Injection treatment (I): radiofrequency denervation of lumbar facet joints at $80^{\circ} \mathrm{C}$ for $90 \mathrm{~s}(n=24)$

Reference treatment $(\mathrm{R})$ : sham radiofrequency denervation with identical procedure except for the radiofrequency lesion $(n=27)$

Outcomes Analyses were split to allow comparisons between those with a good $(n=30)$ or equivocal $(n=11)$ response to facet joint block. Subjects with a good response in the (I) group had significantly lower mean (SE) VAS (34 (6.9) vs. 60 (9.8)) and McGill pain scores $(9(2.3)$ vs. $16(2.8)) 1$ month post-treatment, compared with the (R) group. 6 months post-treatment VAS scores were significantly low in the (I) compared with (R). In subjects with an equivocal response to facet joint block, no significant differences were found between groups at any time-point

Notes Adverse events were not reported

Methodological quality: randomization ?; treatment allocation ?; baseline similarity + ; patients blinded + ; care providers blinded -; outcomes blinded +; co-interventions ?; compliance ?; drop outs +; timing of outcomes +; intention-to-treat + . Total score $=6$

Study: Geurts 2003

Methods RCT; "randomly allocated"

Participants 83 patients, aged 18 years and more, with chronic LBP and a predominance of leg pain who responded positively to lumbar nerve blocks. Exclusion criteria were: previous radiofrequency treatment; indications for surgery (great pain, rapid progressive paresis, cauda equina syndrome); pregnancy; coagulation disorders; malignant disease; allergy to radiopaque contrast or local anaesthetics; and presence of neuropathic sensory or motor deficit, a non-segmental pattern of irradiating leg pain, or both

Three patients required surgery post-treatment and were excluded from the analysis

Interventions Injection treatment (I): radiofrequency lesioning of dorsal root ganglion at $67^{\circ} \mathrm{C}$ for $90 \mathrm{~s}(n=44)$

Reference treatment $(\mathrm{R})$ : sham radiofrequency lesioning of dorsal root ganglion, identical procedure as (I) without the application of radiofrequency current $(n=36)$

Outcomes Three months post-treatment, $16 \%$ of (I) were assessed to have had successful treatment compared with $25 \%$ in (R) group, based on a composite outcome measure (difference $-9.1 \%$ [95\% CI -33.0 to 12.0], $P=0.43$ ). No significant differences between groups were reported for mean change scores (SD) on back pain VAS (I) -0.6 (2.2) versus (R) -1.1 (2.4); change in daily activities (I) -0.5 (3.9) versus (R) -0.4 (3.4); change in analgesics use (I) 0.1 (1.4) versus (R) -0.2 (0.9); global subjective efficacy rating of benefit (I) $21 \%$ versus (R) $17 \%$; or on any SF-36 subscales

Notes $\quad 41$ patients had at least one previous low-back surgery

Adverse events did not differ between treatments, and no serious complications or side effects arose in either group

Methodological quality: randomization + ; treatment allocation + ; baseline similarity + ; patients blinded + ; care providers

blinded +; outcomes blinded +; co-interventions ?; compliance + ; drop outs + ; timing of outcomes + ; intention-to-treat + . Total score $=10$

Study: Kvarstein 2009

Methods RCT; "randomized"

Participants 20 patients aged 20-65 years, with unremitting low-back pain for 6 months. All patients with signs of disc degeneration (MRI) or posterior annular tear (CT) underwent a three-level (L3/L4, L4/L5 and L5/S1) pressure-controlled provocation discography. Patients were eligible if the discography reproduced typical ("concordant") and intensive low-back pain (>7/10) at only one of the three levels. All eligible patients had pain $>5 / 10$ which was exacerbated by sitting and relieved by laying. Exclusion criteria included: positive medial branch blocks, acute infection, history of drug abuse, psychological disturbance, previous spine surgery, abnormal neurological examination, structural deformities or canal stenosis, pregnancy and disc herniations $>4 \mathrm{~mm}$

Interventions Injection treatment (I): percutaneous intradiscal radiofrequency thermocoagulation (PIRFT) at a single level for 10 min (starting at $50^{\circ} \mathrm{C}$, increasing by $5^{\circ} \mathrm{C}$ every $2 \mathrm{~min}$, and ending with 4 min at $\left.65^{\circ} \mathrm{C}\right)(n=10)$

Reference treatment $(\mathrm{R})$ : sham percutaneous intradiscal radiofrequency thermocoagulation (PIRFT), same procedure as above but the annulus was not exposed to RF heating $(n=10)$ 
Table 3 continued

Outcomes After 6 months, there were no significant differences between (I) and (R) on pain intensity. Mean (SD) pain intensity at baseline, 6 and 12 months: (I) 5.4 (2.1), 4.4 (2.7), 4.0 (3.0) versus (R) 6.5 (2.2), 6.5 (2.0), 4.9 (2.7), respectively. No significant differences were observed between groups on any of the secondary outcome measures

Notes Any serious adverse effects attributable to the treatment were not reported

Methodological quality: randomization + ; treatment allocation + ; baseline similarity + ; patients blinded + ; care providers

blinded - ; outcomes blinded + ; co-interventions - ; compliance - ; drop outs + ; timing of outcomes + ; intention-to-treat + . Total score $=8$

Study: Leclaire 2001

Methods RCT; "randomization was performed in blocks of four"

Participants 70 patients, aged 18-65 years, with chronic LBP and positive lumbar facet joint block. Exclusion criteria included allergy to local anaesthetic, blood coagulation disorder, cardiac pacemaker, sciatic pain with neurological deficit, specific low-back pain and previous low-back surgery

Interventions Injection treatment (I): radiofrequency facet joint denervation under fluoroscopic guidance, $80^{\circ} \mathrm{C}$ for $90 \mathrm{~s}$ at a minimum of two levels $(n=36)$

Reference treatment $(\mathrm{R})$ : sham radiofrequency denervation, same procedure as $(\mathrm{I})$, without radiofrequency lesion $(n=34)$

Outcomes $\quad$ After 4 weeks, mean (SD) Roland-Morris scores improved by 8.4 (17.4) in the (I) group and by 2.2 (14.7) in (R), showing a mean difference $(95 \% \mathrm{CI})$ of $6.2(-1.3$ to 13.8$)$. There was no significant treatment effect after 12 weeks. No significant differences were found at any time point for pain VAS, Oswestry score, or the secondary outcomes

Notes No complications after the intervention was reported by patients

Methodological quality: randomization + ; treatment allocation + ; baseline similarity + ; patients blinded + ; care providers blinded -; outcomes blinded + ; co-interventions + ; compliance + ; drop outs + ; timing of outcomes + ; intention-to-treat + . Total score $=10$

Study: Lierz 2004

Methods RCT; randomization performed by computer

Participants 40 patients, aged 20-70 years, with chronic LBP. Exclusion criteria included presence of a motor deficit, recent change of analgesics, pulmonary hypertension, pulmonary artery stenosis, aortic stenosis, mitral stenosis, AV-block grade III, extreme hypovolaemia, coagulation disorders, pregnancy or sepsis/infection

Interventions Injection treatment (I): lumbar epidural block (10 $\mathrm{ml}$ ropivacaine $0.2 \%)(n=20)$

Injection treatment (I2): lumbar epidural block (10 ml bupivacaine 1.125\%) $(n=20)$

Eight single shot epidural blocks at an interval of 2 or 3 days. Active physiotherapy was performed immediately after each session in both groups

Outcomes There was no significant difference between groups in analgesia post-treatment. Mean (SD) pain intensity in (I) decreased from 7.0 (1.6) to 4.0 and from 7.1 (1.6) in (I2) to 4.2. No significant differences between groups were reported in haemodynamic variables

Notes There were three cases of short episodes of headache, two in the (I) group and one in the (I2) group.

Methodological quality: randomization + ; treatment allocation ?; baseline similarity + ; patients blinded + ; care providers blinded +; outcomes blinded ?; co-interventions +; compliance +; drop outs +; timing of outcomes + ; intention-to-treat - . Total score $=8$

Study: Lilius 1989

Methods RCT; randomisation method not stated

Participants 109 patients aged 19-64 years with chronic LBP

Interventions Injection treatment (I): lumbar facet joint injection of corticosteroid and local anaesthetic [6 $\mathrm{ml}$ (30 mg) bupivacaine hydrochloride mixed with $2 \mathrm{ml}(80 \mathrm{mg})$ methylprednisolone acetate] $(n=28)$

Injection treatment (I2): the same mixture, injected peri-capsularly around the lumbar facet joint $(n=39)$

Reference treatment $(\mathrm{R})$ : sham lumbar facet joint injection ( $8 \mathrm{ml}$ of physiological saline) $(n=42)$

Outcomes No significant differences between groups was seen post-treatment, after 2 weeks, or after 6 weeks in work status, pain intensity, or physical range of motion measurements. Data in graphs

Notes Twenty-seven patients had a history of previous vertebral disc surgery

Few side effects were reported and their occurrence did not differ between groups

Methodological quality: randomization ?; treatment allocation -; baseline similarity ?; patients blinded -; care providers blinded -; outcomes blinded -; co-interventions ?; compliance +; drop outs +; timing of outcomes + ; intention-to-treat - . Total score $=3$

Study: Manchikanti 2001

Methods RCT; randomization procedure not described 
Table 3 continued

Participants 73 patients with LBP with or without lower extremity pain for more than 6 months who did not exhibit neurological deficits and who responded positively to lidocaine blocks

Interventions Injection treatment (I): lumbar facet joint injection with local anaesthetic and Sarapin $(0.5 \%$ lidocaine or $0.25 \%$ bupivacaine mixed with equal volumes of Sarapin) $(n=32)$

Injection treatment (I2): lumbar facet joint injection with a mixture of local anaesthetic, Sarapin, and corticosteroid $(0.5 \%$ lidocaine or $0.25 \%$ bupivacaine mixed with equal volumes of Sarapin and $1 \mathrm{mg}$ of methyl prednisolone/ml of the mixture) $(n=41)$

Outcomes Results showed that patients underwent multiple procedures over a period of $2 \frac{1}{2}$ years. Post-treatment, no significant differences were reported between the groups in pain relief, overall health status, physical, functional or psychological status. Average pain scores (mean $\pm \mathrm{SEM}$ ) decreased from $7.6 \pm 0.13$ to $3.5 \pm 0.26$ in (I) and from $7.7 \pm 0.12$ to $3.3 \pm 0.15$ in (I2)

Notes $\quad 17$ patients had previous lumbar laminectomy

No complications were reported in any of the patients

Methodological quality: randomization ?; treatment allocation -; baseline similarity +; patients blinded - ; care providers

blinded -; outcomes blinded ?; co-interventions -; compliance +; drop outs +; timing of outcomes - ; intention-to-treat - . Total score $=3$

Study: Manchikanti 2008

Methods RCT; "computer-generated random allocation sequence"

Participants 120 patients aged over 18 years with chronic non-specific LBP and a diagnosis of lumbar facet joint pain by lumbar anaesthetic block. Exclusion criteria were lack of positive response to controlled comparative local anaesthetic blocks, uncontrollable or unstable opioid use, psychiatric disorders, pregnancy, and patients with a history or potential for adverse reaction(s) to local anaesthetic, Sarapin or steroid

Interventions Injection treatment (I): lumbar facet joint nerve blocks with local anaesthetic (bupivacaine $0.25 \%)(n=60)$

Injection treatment (I2): lumbar facet joint nerve blocks with a mixture of bupivacaine and betamethasone $(n=60)$

Outcomes There were no significant differences in treatment effect of (I) compared to (I2) at 3, 6, or 12 months post-treatment. Pain scores (mean $\pm \mathrm{SD}$ ) at baseline: (I) $8.2 \pm 0.8$, (I2) $7.9 \pm 1.0$; at 3 months (I) $3.8 \pm 1.3$, (I2) $3.5 \pm 1.1$; at 6 months (I) $3.6 \pm 1.5$, (I2) $3.3 \pm 0.8$; and at 12 months (I) $3.7 \pm 1.7$, (I2) $3.5 \pm 1.1$

Notes There were no major adverse events reported over a period of 1 year

Methodological quality: randomization + ; treatment allocation + ; baseline similarity + ; patients blinded + ; care providers blinded -; outcomes blinded ?; co-interventions -; compliance + ; drop outs + ; timing of outcomes + ; intention-to-treat + . Total score $=8$

Study: Marks 1992

Methods RCT; "random number system"

Participants 86 patients with chronic LBP present most of the time for at least 6 months. Exclusion criteria were a radicular pattern in either lower limb, straight leg raising limited at $<60^{\circ}$, and evidence of any progressive spinal disorder of non-degenerative origin

Interventions Injection treatment (I): lumbar facet joint injection with corticosteroids and anaesthetic (20 mg methylprednisolone acetate followed by $1.5 \mathrm{ml}$ lignocaine) $(n=42)$

Injection treatment (I2): lumbar facet nerve block of the medial articular branch of the posterior primary ramus from L1 to L5 (20 mg methylprednisolone acetate followed by $1.5 \mathrm{ml}$ lignocaine) $(n=44)$

Outcomes (I) was slightly better in relieving pain than (I2). This difference reached statistical significance only at 1 month $(P<0.005)$ but not immediately after treatment, at 2 weeks, or at 3 months. 1 month post-treatment, $65.9 \%(n=29)$ of (I2) reported no change in pain severity compared to $42.9 \%(n=18)$ in (I)

Notes $\quad 5$ subjects in each group had previous lumbar spine surgery

No serious complications were reported. Transient symptoms, such as headache, paraesthesia of one leg, nausea and worsening of pain occurred 15 times in (I) and 18 times in (I2)

Methodological quality: randomization + ; treatment allocation + ; baseline similarity + ; patients blinded + ; care providers

blinded ?; outcomes blinded +; co-interventions -; compliance + ; drop outs + ; timing of outcomes + ; intention-to-treat - . Total score $=8$

Study: Mauro 2000

Methods RCT; randomization procedure not described

Participants 60 patients, aged 18-65 years, with chronic LBP or sciatic neuritis of mechanical origin for at least 6 months, and a pain intensity of at least $60 \mathrm{~mm}$ on a VAS. Exclusion criteria included pregnancy, severe concurrent illnesses, and intolerance to paracetamol

Interventions Injection treatment (I): intramuscular injection (2 $\mathrm{ml}$ ampoules containing 1,000 mg vitamin B12) once daily for a 2-week period $(n=30)$

Reference treatment $(\mathrm{R})$ : sham intramuscular injection $(2 \mathrm{ml}$ placebo ampoules) once daily for a 2-week period $(n=30)$ 
Table 3 continued

Outcomes At the end of the treatment period, pain VAS scores (mean \pm SD) significantly decreased in $(\mathrm{I})(75.53 \pm 8.9$ to $9.53 \pm 16.5)$ compared with $(\mathrm{R})(70.63 \pm 7.9$ to $36.83 \pm 27.4)(P<0.0001)$. Total scores on RDQ (mean $\pm \mathrm{SD})$ significantly declined more in (I) (from $13.27 \pm 2.7$ to $2.43 \pm 2.6$ ) than in (R) (from $11.53 \pm 2.2$ to $5.80 \pm 3.3)(P<0.0002)$. Mean consumption of paracetamol over the treatment period was higher in $(\mathrm{R})$ compared to (I) $(P<0.01)$

Notes $\quad$ No changes in vital signs or adverse effects were noted

Methodological quality: randomization ?; treatment allocation ?; baseline similarity -; patients blinded ?; care providers blinded ?; outcomes blinded +; co-interventions ?; compliance +; drop outs -; timing of outcomes +; intention-to-treat - . Total score $=3$

Study: Mayer 2004

Methods RCT; assignment based on date of the month of their initial visit

Participants 70 patients with chronic disabling work-related lumbar spine disorders who had lumbar rigidity between 1 and 3 levels. Exclusion criteria included failure to achieve insurance pre-authorization and more than three levels of spinal rigidity

Interventions Injection treatment (I): lumbar facet joint injection on one to three levels bilaterally ( $1 \mathrm{ml} 2 \%$ lidocaine, $1 \mathrm{ml} 0.5 \%$ bupivacaine, and $1 \mathrm{ml}$ of a depot corticosteroid preparation). In addition, a home stretching exercise program was instructed $(n=36)$

Reference treatment $(\mathrm{R})$ : home stretching exercise program $(n=34)$

Outcomes No significant differences were found in pain and disability between the groups. The (I) group had a significantly greater improvement in range of motion at 5-7 weeks compared withh (R). Pain intensity (mean \pm SD) improved from $6.3 \pm 1.5$ to $5.4 \pm 1.6$ in $(\mathrm{I})$ and $6.7 \pm 1.8$ to $5.9 \pm 2.1$ in $(\mathrm{R})$

Notes $\quad 18$ patients had a history of lumbar spinal surgery

Adverse events were not reported by the study authors

Methodological quality: randomization -; treatment allocation -; baseline similarity + ; patients blinded - ; care providers

blinded -; outcomes blinded +; co-interventions ?; compliance ?; drop outs +; timing of outcomes ?; intention-to-treat ?. Total score $=3$

Study: Nath 2008

Methods RCT; "computer generated randomization schedule"

Participants 40 patients with chronic LBP of at least 2 years duration with $80 \%$ pain relief from two successive medial branch blocks. Excluded were patients with pregnancy, coagulopathies, malignancy, infections, mental handicap and psychiatric disorders; patients with a motor deficit or any other indication for surgical treatment

Interventions Injection treatment (I): radiofrequency denervation of lumbar facet joint for $60 \mathrm{~s}$ at $85^{\circ} \mathrm{C}(n=20)$

Reference treatment (R): sham radiofrequency denervation of lumbar facet joint, same procedure as (I) but without application of current $(n=20)$

Outcomes At baseline, (I) had significantly more generalised pain, low-back pain, hip ROM, and referred pain when compared with (R). 6 months post-treatment, on a 6-point global assessment scale, the (I) group had significantly more than the (R) group by 0.8 points $(P<0.004)$. Mean low-back pain VAS improved from 6.03 to 4.10 in $(\mathrm{I})$ and from 4.35 to 3.98 in $(\mathrm{R})(P=0.02)$. In various secondary outcome measures, the (I) group exhibited improvements that were statistically and clinically greater than those in the $(\mathrm{R})$ group

Notes Adverse events were not reported

Methodological quality: randomization + ; treatment allocation ?; baseline similarity - ; patients blinded + ; care providers blinded +; outcomes blinded +; co-interventions ?; compliance ?; drop outs +; timing of outcomes + ; intention-to-treat ?. Total score $=6$

Study: Oh 2004

Methods RCT; "randomly allocated"

Participants 49 patients with chronic discogenic LBP whose pain continued after undergoing intradiscal electrothermal annuloplasty (IDET) and who also had a positive diagnostic block of the ramus communicans nerve. Exclusion criteria were: spinal stenosis, spinal instability, multilevel ( 2 or more) disc lesions, previous spinal surgery, history of excessive bleeding or coagulopathy and obvious psychologic problems. Patients with a positive diagnostic block of the facet joints were also excluded

Interventions Injection treatment (I): radiofrequency thermocoagulation of the ramus communicans nerve (1\% lidocaine was injected followed by $\mathrm{RF}$ thermocoagulation) at $65^{\circ} \mathrm{C}$ for $60 \mathrm{~s}(n=26)$

Reference treatment $(\mathrm{R})$ : sham radiofrequency thermocoagulation, same procedure as (I), injection of $2 \mathrm{ml}$ of preservative-free $1 \%$ lidocaine without RF thermocoagulation $(n=23)$

Outcomes $\quad$ After 4 months, VAS pain scores were significantly lower in the (I) group than in the (R) group $(P<0.05)$. The scores of the (I) group were a mean of 11.3 points higher $(P<0.05)$ on the bodily pain subscale, and a mean of 12.4 points higher $(P<0.05)$ on the SF-36 physical function subscale, compared with the (R) group 
Table 3 continued

Notes One patient in the (I) group complained of mild lower limb dysesthesia and weakness

Methodological quality: randomization ?; treatment allocation -; baseline similarity +; patients blinded ?; care providers

blinded -; outcomes blinded ?; co-interventions ?; compliance +; drop outs ?; timing of outcomes +; intention-to-treat ?.

Total score $=3$

Study: Pauza 2001

Methods RCT; "computer-generated, random numbers"

Participants 64 patients aged 18-65 years with chronic LBP greater than leg pain and a posterior tear of the annulus fibrosus on provocation discography. Exclusion criteria were previous lumbar spine surgery; abnormal neurological examination; radicular pain; structural deformities; large or sequestered disc herniations; cervical or thoracic pain; uncontrolled or acute medical illnesses; rheumatoid arthritis; ambulatory dysfunction; pregnancy; workman's compensation; injury litigation; disability remuneration; and allergy to contrast media or drugs to be used in the intended procedure

Eight patients violated the protocol and were excluded from the final analysis

Interventions Injection treatment (I): intradiscal electrothermal therapy (IDET) to $90^{\circ} \mathrm{C}$ and a 12-week post-operative rehabilitation program (lumbar corset for 6 weeks, spine stabilization exercises for 6 weeks) $(n=32)$

Reference treatment $(\mathrm{R})$ : sham intradiscal electrothermal therapy, same procedure as above without application of current, and a 12-week post-operative rehabilitation program $(n=24)$

Outcomes 6 months post-treatment, pain VAS scores (mean \pm SD) in the (I) group decreased significantly more $(6.6 \pm 1.4$ to $4.2 \pm 2.6)$ than the $(\mathrm{R})$ group $(6.5 \pm 1.9$ to $5.4 \pm 2.7)(P<0.05)$. Oswestry disability scores (mean $\pm \mathrm{SD})$ decreased from $31 \pm 10$ to $20 \pm 12$ in (I) and from $33 \pm 11$ to $28 \pm 15$ in $(\mathrm{R})(P=0.05)$

Notes $\quad$ No patient had any adverse effects attributable to their treatment

Methodological quality: randomization + ; treatment allocation + ; baseline similarity + ; patients blinded + ; care providers

blinded - ; outcomes blinded + ; co-interventions + ; compliance + ; drop outs + ; timing of outcomes + ; intention-to-treat - .

Total score $=9$

Study: Revel 1998

Methods RCT; randomisation method not stated

Participants 80 patients aged 18 years or more with chronic LBP unrelieved by analgesics, NSAIDs, and physical therapy. Exclusion criteria included previous back surgery, sciatica, sacroiliac joint pain, pregnancy, psychiatric disease and a history of adverse reaction to a lidocaine or corticosteroid injection

Interventions Injection treatment (I): lumbar facet joint injection with anaesthetic ( $1 \mathrm{ml} 2 \%$ lidocaine) $(n=42)$

Reference treatment $(\mathrm{R})$ : lumbar facet joint injection with $1 \mathrm{ml}$ saline $(n=38)$

Patients had 4-6 lower joints injected on both sides or 2-3 on the same side

Outcomes Post-treatment, pain relief was significantly better in the (I) group compared with the (R) group. In patients with fewer pain characteristics, pain (mean $\pm \mathrm{SD}$ ) decreased by $-35.36 \pm 23.6$ in (I) compared to $-11.87 \pm 17.44$ in $(\mathrm{R})(P=0.01)$

Notes Adverse events were not reported

Methodological quality: randomization ?; treatment allocation ?; baseline similarity + ; patients blinded + ; care providers blinded +; outcomes blinded +; co-interventions ?; compliance ?; drop outs + ; timing of outcomes + ; intention-to-treat + . Total score $=7$

Study: Serrao 1992

Methods RCT; "randomly allocated"

Participants 28 patients attending chronic LBP treatment. Patients with disc lesions and spinal claudication were excluded

Interventions Injection treatment (I): lumbar epidural injection with corticosteroid (80 $\mathrm{mg}$ prednisolone) plus $3 \mathrm{ml} 5 \%$ dextrose injected into the intrathecal space $(n=14)$

Injection treatment (I2): lumbar epidural injection ( $10 \mathrm{ml}$ normal saline) plus $2 \mathrm{mg}$ midazolam dissolved in $3 \mathrm{ml} 5 \%$ dextrose injected into the intrathecal space $(n=14)$

Outcomes There were no significant differences between the groups post-treatment, or after 2 months follow-up for either pain intensity or unpleasantness. No significant differences were found between groups on psychological measures. Data in graphs

Notes Eight patients in the (I) group and seven in the (I2) group experienced headaches following treatment; nausea was experienced in two of the (I) patients and by one patient in the (R) group

Methodological quality: randomization ?; treatment allocation ?; baseline similarity ?; patients blinded +; care providers blinded ?; outcomes blinded +; co-interventions -; compliance +; drop outs ?; timing of outcomes +; intention-to-treat ?. Total

score $=4$

Study: Tekin 2007

Methods RCT; "random number generation" 
Table 3 continued

Participants 60 patients, aged $>17$ years, with chronic LBP with or without radiating pain into the upper leg, focal tenderness over the facet joints and pain on hyperextension. Exclusion criteria were prior radiofrequency treatment, coagulation disturbances, allergies to contrast media or local anaesthetics, malignancy, psychiatric conditions and pregnancy. Only patients with a positive response ( $>50 \%$ pain relief for sufficient duration) to a diagnostic medial branch block were included

Interventions Injection treatment (I): radiofrequency denervation (conventional) medial branch lumbar nerve roots at $80^{\circ} \mathrm{C}$ for $90 \mathrm{~s}(n=20)$ Injection treatment (I2): radiofrequency denervation (pulsed) medial branch lumbar nerve roots at $42^{\circ} \mathrm{C}$ for $240 \mathrm{~s}(n=20)$

Reference treatment (R): sham radiofrequency denervation, similar procedure as (I), but only bupivacaine $0.5 \% 0.3 \mathrm{ml}$ was injected $(n=20)$

Outcomes Post-treatment VAS and ODI scores (mean \pm SD) were lower in (I) $(2.3 \pm 1.4 ; 25.6 \pm 6.5)$ and (I2) $(2.8 \pm 1.5 ; 24.4 \pm 5.7)$ than in $(\mathrm{R})(4.3 \pm 1.0 ; 30.5 \pm 5.7)(P<0.001)$. At 6 months and 1 year follow-up, mean VAS score was similar in $(\mathrm{R})$ and (I2) groups but lower in the (I) group compared with the others $(P<0.05)$. Mean ODI was lower in (I) and (I2) groups than (R) group at 1 year $(P<0.05)$

Patient satisfaction was lower in $(\mathrm{R})$ than other groups $(P=0.03)$, and highest in $(\mathrm{I})(P=0.004)$

Notes There were no complications related to the procedure during the follow-up period

Methodological quality: randomization +; treatment allocation ?; baseline similarity +; patients blinded +; care providers blinded -; outcomes blinded ?; co-interventions +; compliance +; drop outs +; timing of outcomes +; intention-to-treat ?. Total score $=7$

Study: van Alphen 1989

Methods RCT; randomization method not stated

Participants 151 patients, aged 18-45 years, with proven disc herniation on myelography. 68 patients had symptoms for $>6$ months. Exclusion criteria were severe neurological deficits, previous lumbar disc surgery or chemonucleolysis, and pregnancy

Interventions Injection treatment (I): chemonucleolysis [2 $\mathrm{ml}(4,000 \mathrm{U})$ chymopapain injected into intervertebral disc] followed by physiotherapy $(n=34)$

Reference treatment $(\mathrm{R})$ : discectomy under general anaesthetic using an interlaminar approach followed by post-operative physiotherapy $(n=34)$

Outcomes 12 months post-treatment, status of pain (disappeared, improved, unchanged, increased) $=(\mathrm{I}): n=13,14,4,0 ;(\mathrm{R}): n=16$, $13,5,0$ (not significant)

Notes Complications in both groups included increased neurological symptoms, dural defect with leakage of cerebrospinal fluid, and partial cauda equina in one patient

Methodological quality: randomization + ; treatment allocation - ; baseline similarity ?; patients blinded - ; care providers

blinded -; outcomes blinded -; co-interventions ?; compliance - ; drop outs - ; timing of outcomes + ; intention-to-treat - . Total score $=2$

Study: van Kleef 1999

Methods RCT; "[randomized] with help of a computer program"

Participants 31 patients, aged 20-60 years, with chronic LBP for $>12$ months and a positive diagnostic dorsal ramus nerve block. Patients with previous back surgery or known specific LBP were excluded. One patient refused follow-up and was excluded from the analysis

Interventions Injection treatment (I): radiofrequency denervation of lumbar facet joints (posterior primary ramus of the segmental nerves L3L5) at $80^{\circ} \mathrm{C}$ for $60 \mathrm{~s}$ on one or both sides $(n=15)$

Reference treatment $(\mathrm{R})$ : sham radiofrequency denervation, electrodes introduced as in (I) but no radiofrequency current was applied $(n=16)$

Outcomes After 8 weeks, the mean VAS \pm SD for (R) and (I) was $4.8 \pm 2.5$ and $2.8 \pm 2.4$, respectively. This difference, and the difference in effect on the Oswestry disability scale and globally perceived effect were statistically significant. Three, 6 , and 12 months after treatment there were significantly more "success" patients in the (I) group compared with (R)

Notes There were no complications during or after the interventions

Methodological quality: randomization +; treatment allocation ?; baseline similarity +; patients blinded + ; care providers blinded +; outcomes blinded +; co-interventions ?; compliance +; drop outs ?; timing of outcomes +; intention-to-treat ?. Total score $=7$

Study: van Wijk 2005

Methods RCT; randomisation method not stated

Participants 81 patients, aged 17 years and over, with chronic LBP and a 50\% pain VAS reduction on lumbar facet joint diagnostic block. Exclusion criteria were prior radiofrequency treatment, radicular syndrome, coagulopathies, specific allergies, cancer and pregnancy

Interventions Injection treatment (I): radiofrequency lumbar facet denervation (dorsal ramus medial branches) at $80^{\circ} \mathrm{C}$ for $60 \mathrm{~s}(n=40)$

Reference treatment $(\mathrm{R})$ : sham radiofrequency denervation, same procedure as (I) but without applying radiofrequency current $(n=41)$ 
Table 3 continued

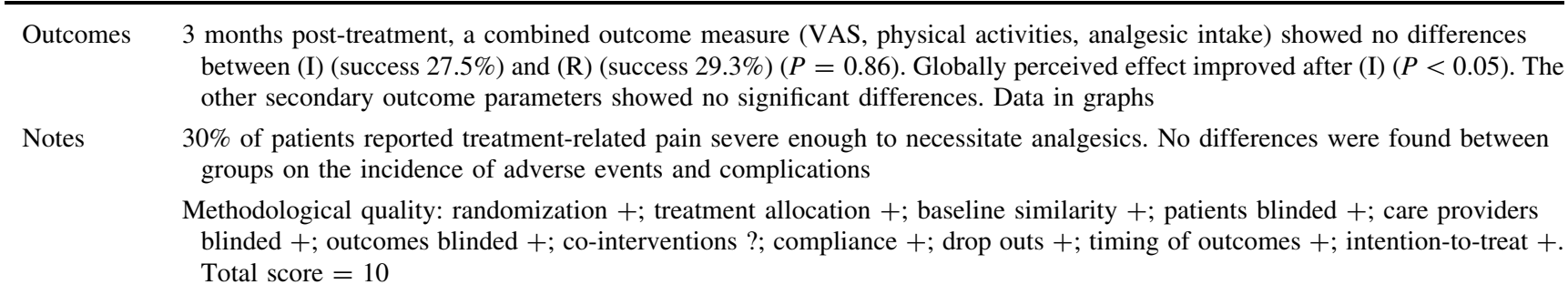

\section{Appendix 2}

See Table 4.

Table 4 Characteristics of excluded studies

\begin{tabular}{|c|c|c|}
\hline Review & Study & Reason for exclusion \\
\hline \multirow[t]{8}{*}{ Staal 2008} & Aldrete 2003 & Duration of LBP not reported \\
\hline & Beliveau 1971 & Duration of LBP not reported \\
\hline & Breivik 1976 & Duration of LBP not reported \\
\hline & Collee 1991 & Duration of LBP not reported \\
\hline & Garvey 1989 & Included patients with duration $<3$ months \\
\hline & Rocco 1989 & All patients had previous laminectomy \\
\hline & Sonne 1985 & Included patients with duration $<3$ months \\
\hline & Takada 2005 & Included patients with duration $<3$ months \\
\hline \multirow[t]{17}{*}{ Gibson $2007^{\mathrm{a}}$} & Schwetschenau 1976 & Included patients with duration $<3$ months \\
\hline & Fraser 1982 & Included patients with duration $<3$ months \\
\hline & Javid 1983 & Included patients with duration $<3$ months \\
\hline & Feldman 1986 & Duration of LBP not reported \\
\hline & Dabezies 1988 & Duration of LBP not reported \\
\hline & Ejeskar 1983 & Duration of LBP not reported \\
\hline & Crawshaw 1984 & Duration of LBP not reported \\
\hline & Lavignolle 1987 & Duration of LBP not reported \\
\hline & Muralikuttan 1992 & Included patients with duration $<3$ months \\
\hline & Benoist 1993 & Duration of LBP not reported \\
\hline & Hedtmann 1992 & Duration of LBP not reported \\
\hline & Bourgeois 1988 & Included patients with duration $<3$ months \\
\hline & Bontoux 1990 & Included patients with duration $<3$ months \\
\hline & Bromley 1984 & Included patients with duration $<3$ months \\
\hline & Yu 1996 & Article in Chinese/Mandarin \\
\hline & Steffen 1996 & Included patients with duration $<3$ months \\
\hline & Revel 1993 & Included patients with duration $<3$ months \\
\hline \multirow[t]{8}{*}{ Abdi 2007} & Wilson-McDonald 2005 & Duration of LBP not reported \\
\hline & Arden 2005 & Included patients with duration $<3$ months \\
\hline & Carette 1997 & Included patients with duration $<3$ months \\
\hline & McGregor 2001 & Duration of LBP not reported \\
\hline & Pirbudak 2003 & No comparisons with injection therapy \\
\hline & Snoek 1977 & Included patients with duration $<3$ months \\
\hline & Cuckler 1985 & Duration of LBP not reported \\
\hline & Dilke 1973 & Included patients with duration $<3$ months \\
\hline
\end{tabular}


Table 4 continued

\begin{tabular}{|c|c|c|}
\hline Review & Study & Reason for exclusion \\
\hline & Ridley 1988 & Duration of LBP not reported \\
\hline & Rogers 1992 & Duration of LBP not reported \\
\hline & Kraemer 1997 & Duration of LBP not reported \\
\hline & Riew 2006 & Duration of LBP not reported \\
\hline & $\mathrm{Ng} 2005$ & Duration of LBP not reported \\
\hline & Karppinen 2001 & Included patients with duration $<3$ months \\
\hline & Vad 2002 & Included patients with duration $<3$ months \\
\hline & Devulder 1999 & Duration of LBP not reported \\
\hline & Thomas 2003 & Included patients with duration $<3$ months \\
\hline & Bush 1991 & Included patients with duration $<3$ months \\
\hline & Matthews 1987 & Included patients with duration $<3$ months \\
\hline & Helsa 1979 & Article in Norwegian \\
\hline & Meadeb 2001 & Included patients with duration $<3$ months \\
\hline \multirow[t]{33}{*}{ Chou 2009} & Hameroff 1981 & Duration of LBP not reported \\
\hline & Dechow 1999 & Outside the scope of this review (prolotherapy) \\
\hline & Klein 1993 & Outside the scope of this review (prolotherapy) \\
\hline & Ongley 1987 & Outside the scope of this review (prolotherapy) \\
\hline & Yelland 2004 & Outside the scope of this review (prolotherapy) \\
\hline & Ackerman 2007 & Included patients with duration $<3$ months \\
\hline & Bonetti 2005 & Included patients with duration $<3$ months \\
\hline & Fukusaki 1998 & Included patients with duration $<3$ months \\
\hline & Gallucci 2007 & Included patients with duration $<3$ months \\
\hline & Jeong 2007 & Included patients with duration $<3$ months \\
\hline & Manchikanti 2004 & No injection therapy comparison \\
\hline & Helliwell 1985 & Included patients with duration $<3$ months \\
\hline & Klenerman 1984 & All patients had acute sciatica ( $<6$ weeks duration) \\
\hline & Zahaar 1991 & Included patients with duration $<3$ months \\
\hline & Buchner 2000 & Included patients with duration $<3$ months \\
\hline & Butterman 2004 & Included patients with duration $<3$ months \\
\hline & Kolsi 2000 & Included patients with duration $<3$ months \\
\hline & Pirbudak 2003b & Included patients with duration $<3$ months \\
\hline & Nash 1989 & Duration of LBP not reported \\
\hline & Luukkainen 2002 & Duration of LBP not reported \\
\hline & Buttermann 2004 & Patients randomized to surgery, not injection therapy \\
\hline & Graham 1975 & Included patients with duration $<3$ months \\
\hline & Khot 2004 & Duration of LBP not reported \\
\hline & Simmons 1992 & Duration of LBP not reported \\
\hline & Graham 1976 & Included patients with duration $<3$ months \\
\hline & Hoogland 2006 & All subjects underwent endoscopic discectomy \\
\hline & Wittenberg 2001 & Duration of LBP not reported \\
\hline & Burton 2000 & Duration of LBP not reported \\
\hline & Krugluger 2000 & Duration of LBP not reported \\
\hline & Steffen 1997 & Included patients with duration $<3$ months \\
\hline & Sanders 1999 & No injection therapy comparison \\
\hline & Kumar 2007 & All patients had failed back surgery \\
\hline & North 2005 & All patients had failed back surgery \\
\hline
\end{tabular}

${ }^{a}$ Studies in this review regarding surgery are not listed 


\section{Appendix 3}

See Table 5.

Table 5 Forest plots and GRADE evidence profiles

Analysis 01.01 IDET vs. placebo for chronic non-specific LBP, Change in pain intensity from baseline on SF-36 Bodily Pain Index (100-point). Long term follow up (6 months).

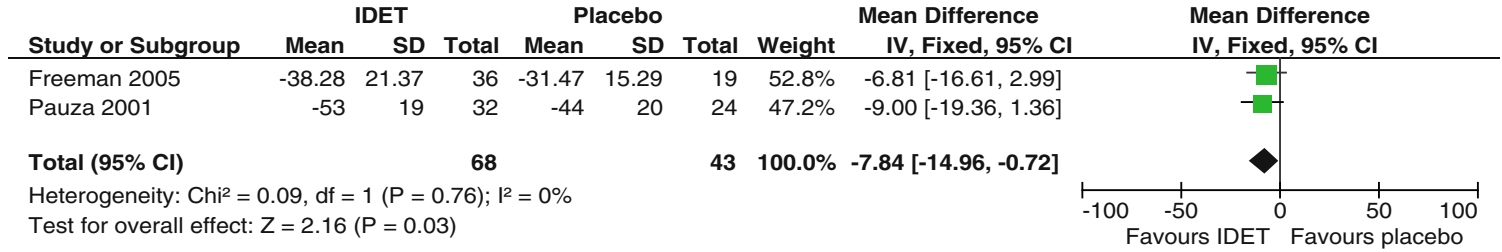

Analysis 01.02 IDET vs. placebo for chronic non-specific LBP, Change in functional status from baseline on Oswestry Disability Index (100-point). Long term follow up (6 months).

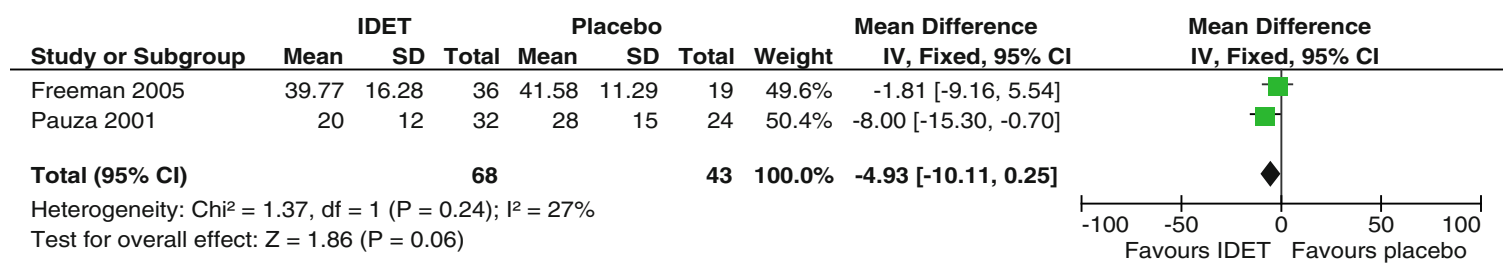

\section{GRADE evidence profile (Analyses 01.01, 01.02)}

Date: 2009-07-01

Question: Should IDET vs. placebo be used for chronic LBP?

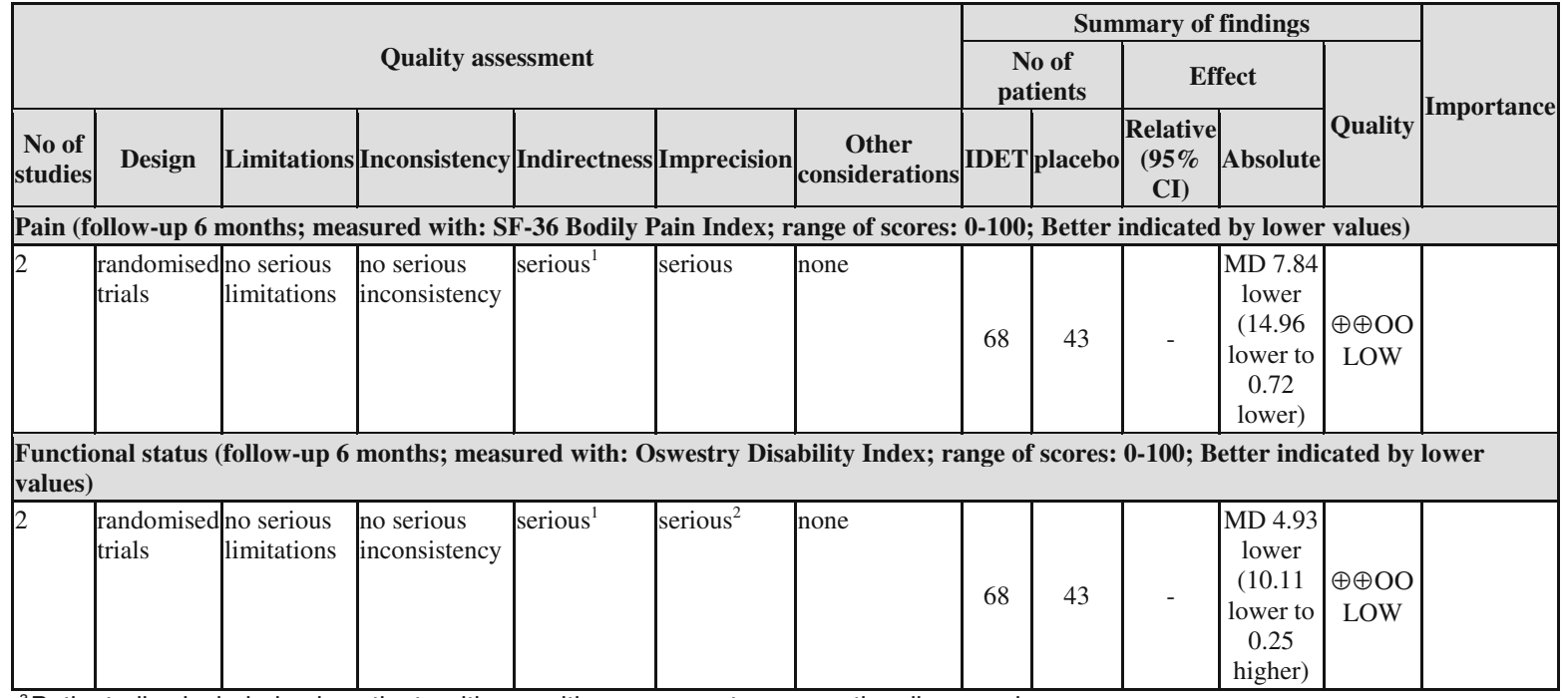

${ }^{a}$ Both studies included only patients with a positive response to provocative discography

${ }^{\mathrm{b}}$ Small sample size 
Table 5 continued

Analysis 02.01 Radiofrequency denervation of facet joints vs. placebo for chronic non-specific LBP, Change in pain intensity from baseline on pain VAS (100-point). Short term follow up ( $\leq 4$ weeks).

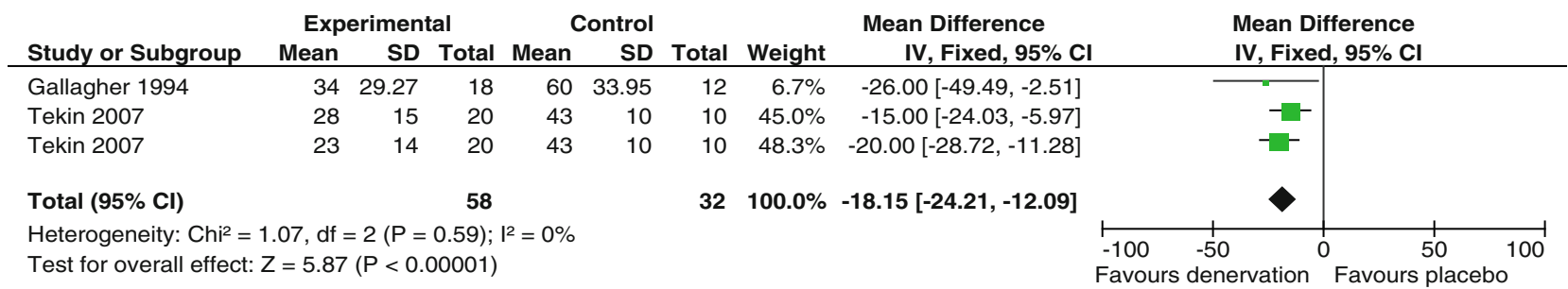

Analysis $\mathbf{0 2 . 0 2}$ Radiofrequency denervation of facet joints vs. placebo for chronic non-specific LBP, Change in pain intensity from baseline on pain VAS (100-point). Intermediate term follow up (1- 6 months).

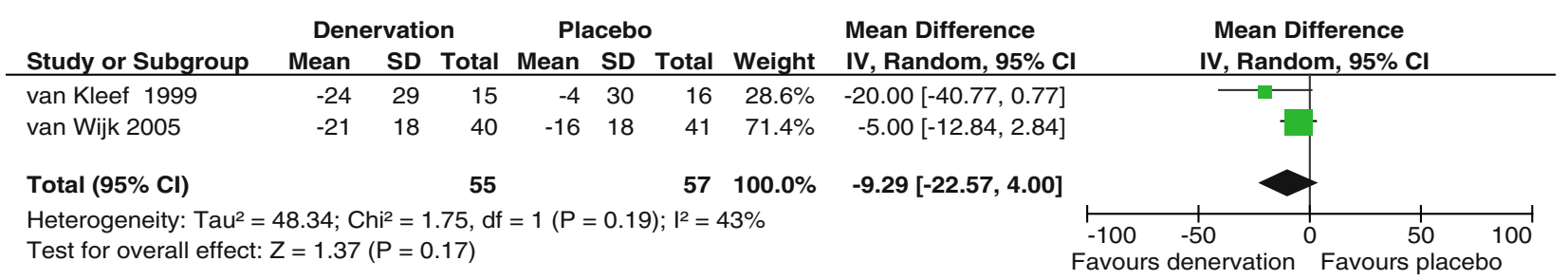

Analysis $\mathbf{0 2 . 0 3}$ Radiofrequency denervation of facet joints vs. placebo for chronic non-specific LBP, Change in pain intensity from baseline on pain VAS (100-point). Long term follow up (6 months).

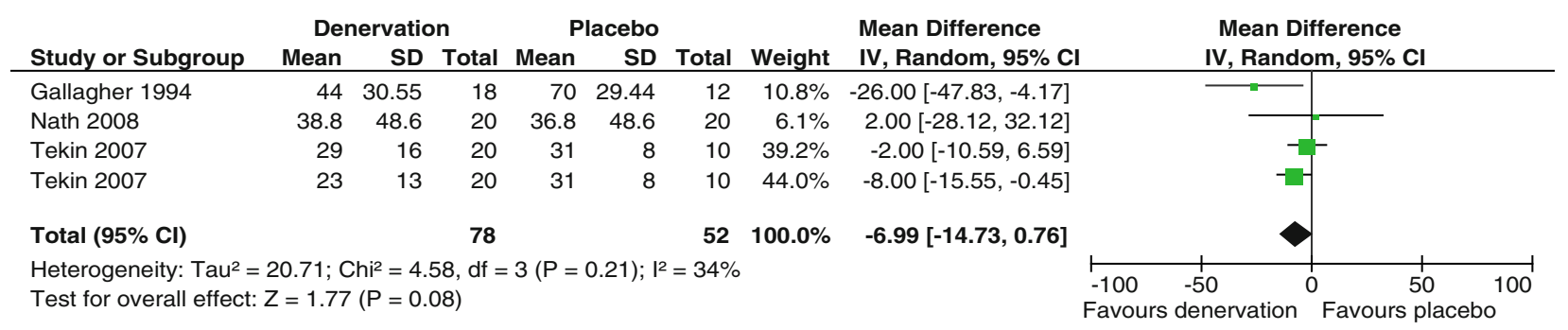

Analysis 02.04 Radiofrequency denervation of facet joints vs. placebo for chronic non-specific LBP, Change in functional status from baseline on Oswestry Disability Index (100-point). Short term follow up ( $\leq 4$ weeks).

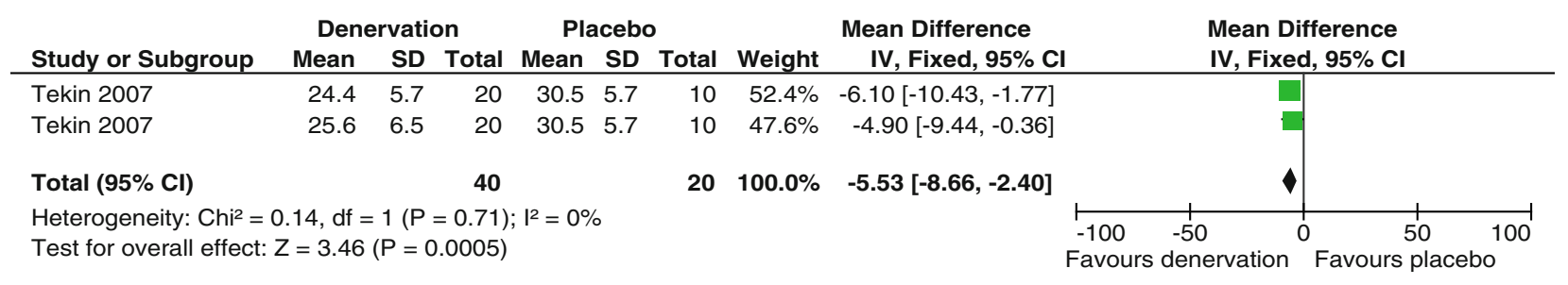


Table 5 continued

GRADE evidence profile (Analyses 02.01, 02.02, 02.03, 02.04)

Date: 2009-07-01

Question: Should radiofrequency denervation of facet joints vs. placebo be used for chronic LBP?

\begin{tabular}{|c|c|c|c|c|c|c|c|c|c|c|c|c|}
\hline \multirow{2}{*}{\multicolumn{7}{|c|}{ Quality assessment }} & \multicolumn{5}{|c|}{ Summary of findings } & \multirow[b]{3}{*}{ Importance } \\
\hline & & & & & & & \multicolumn{2}{|c|}{ No of patients } & \multicolumn{2}{|c|}{ Effect } & \multirow[b]{2}{*}{ Quality } & \\
\hline $\begin{array}{l}\text { No of } \\
\text { studies }\end{array}$ & Design & Limitations & Inconsistency & Indirectness & Imprecision & $\begin{array}{c}\text { Other } \\
\text { considerations }\end{array}$ & denervation & placebo & \begin{tabular}{|c|} 
Relative \\
(95\% \\
CI)
\end{tabular} & Absolute & & \\
\hline \multicolumn{13}{|c|}{ Pain (short term) (follow-up < 4 weeks; measured with: pain VAS; range of scores: 0-100; Better indicated by lower values) } \\
\hline 2 & \begin{tabular}{|l|}
$\begin{array}{l}\text { randomised } \\
\text { trials }\end{array}$ \\
\end{tabular} & \begin{tabular}{|l} 
no serious \\
limitations
\end{tabular} & \begin{tabular}{|l|} 
no serious \\
inconsistency
\end{tabular} & serious $^{1}$ & serious $^{2}$ & none & 58 & 32 & - & \begin{tabular}{|c|} 
MD \\
18.15 \\
lower \\
$(24.21$ to \\
12.09 \\
lower $)$
\end{tabular} & $\begin{array}{c}\oplus \oplus \mathrm{OO} \\
\text { LOW }\end{array}$ & \\
\hline \multicolumn{13}{|c|}{ Pain (intermediate term) (follow-up 1-6 months; measured with: pain VAS; range of scores: 0-100; Better indicated by lower values) } \\
\hline 2 & $\begin{array}{l}\text { randomised } \\
\text { trials }\end{array}$ & $\begin{array}{l}\text { no serious } \\
\text { limitations }\end{array}$ & \begin{tabular}{|l|} 
no serious \\
inconsistency
\end{tabular} & serious $^{1}$ & serious $^{2}$ & none & 55 & 57 & - & \begin{tabular}{|c|} 
MD 9.29 \\
lower \\
$(22.57$ \\
lower to \\
4.00 \\
higher) \\
\end{tabular} & $\begin{array}{c}\oplus \oplus \mathrm{OO} \\
\text { LOW }\end{array}$ & \\
\hline \multicolumn{13}{|c|}{ Pain (long term) (follow-up 6 months; measured with: pain VAS; range of scores: 0-100; Better indicated by lower values) } \\
\hline 3 & $\begin{array}{l}\text { randomised } \\
\text { trials }\end{array}$ & $\begin{array}{l}\text { no serious } \\
\text { limitations }\end{array}$ & $\begin{array}{l}\text { no serious } \\
\text { inconsistency }\end{array}$ & serious $^{1}$ & serious $^{2}$ & none & 78 & 52 & - & \begin{tabular}{|c|} 
MD 6.99 \\
lower \\
$(14.73$ \\
lower to \\
0.76 \\
higher $)$
\end{tabular} & $\begin{array}{l}\oplus \oplus \mathrm{OO} \\
\text { LOW }\end{array}$ & \\
\hline \multicolumn{13}{|c|}{$\begin{array}{l}\text { Functional status (short term) (follow-up < } 4 \text { weeks; measured with: Oswestry Disability Index; range of scores: 0-100; Better indicated by } \\
\text { lower values) }\end{array}$} \\
\hline 1 & $\begin{array}{l}\text { randomised } \\
\text { trials }\end{array}$ & $\begin{array}{l}\text { no serious } \\
\text { limitations }\end{array}$ & serious $^{3}$ & serious $^{1}$ & serious $^{2}$ & none & 40 & 20 & - & \begin{tabular}{|c|} 
MD 5.53 \\
lower \\
$(8.66$ \\
lower to \\
2.40 \\
higher)
\end{tabular} & $\begin{array}{c}\oplus \mathrm{OOO} \\
\text { VERY } \\
\text { LOW }\end{array}$ & \\
\hline
\end{tabular}

${ }^{a}$ All studies included only patients with a positive response to local anaesthetic nerve block

${ }^{b}$ Small sample size

' Single RCT, by definition is inconsistent

\section{References}

1. Manchikanti L, Singh V, Datta S, Cohen SP, Hirsch JA (2009) Comprehensive review of epidemiology, scope, and impact of spinal pain. Pain Physician 12(4):E35-E70

2. Martin BI, Deyo RA, Mirza SK, Turner JA, Comstock BA, Hollingworth W, Sullivan SD (2008) Expenditures and health status among adults with back and neck problems. JAMA 299(6):656-664

3. Staal JB, de Bie R, de Vet HCW, Hildebrandt J, Nelemans $P$ (2008) Injection therapy for subacute and chronic low-back pain. Cochrane Database Syst Rev (3):Art. No.: CD001824. doi: 10.1002/14651858.CD001824.pub3

4. Abdi S, Datta S, Trescot AM et al (2007) Epidural steroids in the management of chronic spinal pain: a systematic review. Pain Physician 10:185-212

5. Gibson JNA, Waddell G (2007) Surgical interventions for lumbar disc prolapse. Cochrane Database Syst Rev (2):Art. No.: CD001350. doi:10.1002/14651858.CD001350.pub4

6. Chou R, Atlas SJ, Stanos SP, Rosenquist RW (2009) Nonsurgical interventional therapies for low back pain: a review of the evidence for an American Pain Society clinical practice guideline. Spine 34(10):1078-1093

7. Furlan AD, Pennick V, Bombardier C, van Tulder M (2009) Updated method guidelines for systematic reviews in the Cochrane Back Review Group. Spine 34(18):1929-1941
8. van Tulder M, Suttorp M, Morton S, Bouter L, Shekelle P (2009) Empirical evidence of an association between internal validity and effect size in randomized controlled trials of low-back pain. Spine 34(16):1685-1692

9. Atkins D, Best D, Briss PA et al (2004) Grading quality of evidence and strength of recommendations. BMJ 328:1490

10. Guyatt GH, Oxman AD, Vist G et al (2008) Rating quality of evidence and strength of recommendations GRADE: an emerging consensus on rating quality of evidence and strength of recommendations. BMJ 336:924-926

11. van Alphen HA, Braakman R, Bezemer PD et al (1989) Chemonucleolysis or discectomy: a randomized multicenter trial. J Neurosurg 70:869-875

12. Carette S, Marcoux S, Truchon R, Grondin C, Gagnon J, Allard Y et al (1991) A controlled trial of corticosteroid injections into facet joints for chronic low back pain. N Eng J Med 325(14):1002-1007

13. Lilius G, Laasonen EM, Myllynen P, Harilainen A, Gronlund G (1989) Lumbar facet joint syndrome. A randomised clinical trial. J Bone Joint Surg 71:681-684

14. Revel M, Poiraudeau S, Auleley GR, Payan C, Nguyen M, Chevrot A et al (1998) Capacity of the clinical picture to characterize low back pain relieved by facet joint anesthesia. Proposed criteria to identify patients with painful facet joints. Spine 23(18): 1972-1976

15. Mayer TG, Gatchel RJ, Keeley J, McGeary D, Dersh J, Anagnostis C (2004) A randomized clinical trial of treatment for lumbar segmental rigidity. Spine 29(20):2199-2205 
16. Fuchs S, Erbe T, Fischer HL, Tibesku CO (2005) Intraarticular hyaluronic acid versus glucocorticoid injections for nonradicular pain in the lumbar spine. J Vasc Interv Radiol 16(11):1493-1498

17. Manchikanti L, Pampati V, Bakhit C, Rivera JJ, Beyer CD, Damron KS et al (2001) Effectiveness of lumbar facet joint nerve blocks in chronic low back pain: a randomized clinical trial. Pain Physician 4(1):101-107

18. Manchikanti L, Singh V, Falco FJE, Cash KA, Pampati V (2008) Lumbar facet joint nerve blocks in managing chronic facet joint pain: one-year follow-up of a randomized, double-blind controlled trial: clinical trial NCT00355914. Pain Physician 11:121-132

19. Marks RC, Houston T, Thulbourne T (1992) Facet joint injection and facet nerve block: a randomised comparison in 86 patients with chronic low back pain. Pain 49(3):325-328

20. Dashfield AK, Taylor MB, Cleaver JS et al (2005) Comparison of caudal steroid epidural with targeted steroid placement during spinal endoscopy for chronic sciatica: a prospective, randomized, double-blind trial. Br J Anaesth 94:514-519

21. Serrao JM, Marks RL, Morley SJ, Goodchild CS (1992) Intrathecal midazolam for the treatment of chronic mechanical low back pain: a controlled comparison with epidural steroid in a pilot study. Pain 48(1):5-12

22. Lierz P, Gustorff B, Markow G, Felleiter P (2004) Comparison between bupivacaine $0.125 \%$ and ropivacaine $0.2 \%$ for epidural administration to outpatients with chronic low back pain. Eur $\mathbf{J}$ Anaesthesiol 21(1):32-37

23. Mauro GL, Martorana U, Cataldo P, Brancato G, Letizia G (2000) Vitamin B12 in low back pain: a randomised, doubleblind placebo-controlled study. Eur Rev Med Pharmacol Sci 4(3):53-58

24. Foster L, Clapp L, Erickson M et al (2001) Botulinum toxin A and chronic low back pain: a randomized, double-blind study. Neurology 56:1290-1293

25. Barendse GA, van Den Berg SG, Kessels AH et al (2001) Randomized controlled trial of percutaneous intradiscal radiofrequency thermocoagulation for chronic discogenic back pain. Spine 26:287-292

26. Ercelen O, Bulutcu E, Oktenoglu T et al (2003) Radiofrequency lesioning using two different time modalities for the treatment of lumbar discogenic pain: a randomized trial. Spine 28:1922-1927

27. Freeman BJ, Fraser RD, Cain CM et al (2005) A randomized, double-blind, controlled trial: intradiscal electrothermal therapy versus placebo for the treatment of chronic discogenic low back pain. Spine 30:2369-2377

28. Kvarstein G, Måwe L, Indahl A, Hol PC, Tennøe B, Digernes R, Stubhaug A, Tønnessen TI, Beivik H (2009) A randomized double-blind controlled trial of intra-annular radiofrequency thermal disc therapy-a 12-month follow-up. Pain 145:279-286

29. Pauza KJ, Howell S, Dreyfuss P et al (2004) A randomized, placebo-controlled trial of intradiscal electrothermal therapy for the treatment of discogenic low back pain. Spine J 4:27-35

30. Oh WS, Shim JC (2004) A randomized controlled trial of radiofrequency denervation of the ramus communicans nerve for chronic discogenic low back pain. Clin J Pain 20(1):55-60

31. Gallagher J, Petriccione D, Wedley J et al (1994) Radiofrequency facet joint denervation in the treatment of low back pain: a prospective controlled double-blind study to assess its efficacy. Pain Clin 7:193-198

32. Leclaire R, Fortin L, Lambert R et al (2001) Radiofrequency facet joint denervation in the treatment of low back pain: a placebo-controlled clinical trial to assess efficacy. Spine 26:14111416

33. Nath S, Nath C, Pettersson K (2008) Percutaneous lumbar zygapophysial (facet) joint neurotomy using radiofrequency current, in the management of chronic low back pain. Spine 33:12911297

34. Tekin I, Mirzai H, OK G et al (2007) A comparison of conventional and pulsed radiofrequency denervation in the treatment of chronic facet joint pain. Clin J Pain 23:524-529

35. van Kleef M, Barendse G, Kessels A et al (1999) Randomized trial of radiofrequency lumbar facet denervation for chronic low back pain. Spine 24:1937-1942

36. van Wijk R, Geurts J, Wynne H et al (2005) Radiofrequency denervation of lumbar facet joints in the treatment of chronic low back pain: a randomized, double-blind, sham lesion-controlled trial. Clin J Pain 21:335-344

37. Geurts J, van Wijk RM, Wynne HJ et al (2003) Radiofrequency lesioning of dorsal root ganglia for chronic lumbosacral radicular pain: a randomised, double-blind, controlled trial. Lancet 361:21-26

38. Egger M, Smith GD (1998) Meta-analysis bias in location and selection of studies. BMJ 316:61-66 